\title{
Economic Policy Uncertainty and the Yield Curve*
}

\author{
Markus Leippold ${ }^{\dagger} \quad$ Felix H. A. MATthys ${ }^{\ddagger}$
}

October 30,2015

\begin{abstract}
We study the impact of economic policy uncertainty on the term structure of nominal interest rates. We develop a general equilibrium model, in which the real side of the economy is driven by government policy uncertainty and the central bank sets money supply endogenously following a Taylor rule. We analyze the impact of government and monetary policy uncertainty on nominal yields, short rates, bond risk premia, and the term structure of bond yield volatility. Our affine yield curve model is able to capture both the shape of the interest rate term structure as well as the hump-shape of bond yield volatilities. Our empirical analysis shows that higher government policy uncertainty leads to a decline in yields and an increase in bond yield volatility, whereas monetary policy uncertainty has no significant contemporaneous effect on yields nor volatilities. However, it is an important predictor for bond risk premia.
\end{abstract}

JEL classification: G01, G12, G14, G18

Key Words: Term structure modeling, yield volatility curve, policy uncertainty, bond risk premia

\footnotetext{
${ }^{*}$ This paper benefited greatly from discussions with Yacine Aït-Sahalia, Caio Almeida, Markus Brunnermeier, Jérôme Detemple, Itamar Drechsler, Darell Duffie, Fabio Trojani, Valentin Haddad, Oleg Itskhoki, Jakub Jurek, Philippe Mueller, Jean-Charles Rochet, Christopher Sims, David Srear, Adi Sunderam, Josef Teichmann, and Wei Xiong. For helpful comments we would like to thank the seminar participants of the 2015 SAFE Asset Pricing Workshop in Frankfurt, the Finance and Math Seminar ETH and University of Zurich, the 12th Doctorial Workshop in Finance at Gerzensee, the Princeton Student Research Workshop, Financial Mathematics Seminar at ORFE Princeton University, Bank of England, Central Bank of Mexico, and ITAM. Financial support from the Swiss Finance Institute (SFI), Bank Vontobel, the Swiss National Science Foundation and the National Center of Competence in Research "Financial Valuation and Risk Management" is gratefully acknowledged.

${ }^{\dagger}$ Swiss Finance Institute (SFI) and University of Zurich, Department of Banking and Finance, Plattenstrasse 14, 8032 Zurich, Switzerland; markus.leippold@bf .uzh.ch.

${ }^{\ddagger}$ Princeton University, Bendheim Center For Finance; fmatthys@princeton.edu.
} 


\section{Introduction}

Economic policy is driven by government and central banking actions. Governments define fiscal policy and impose regulations, while central banks manage the money supply and set nominal short rates. These policies have a fundamental impact on financial markets. However, despite good intentions their effectiveness remains uncertain at best. In this paper, we explore the impact of such policy uncertainty on the term structure of interest rates, its corresponding volatility curve, and on bond risk premia. We develop a general equilibrium model, in which the real side of the economy is subject to government policy uncertainty and the nominal side of the economy is affected by monetary policy shocks. Our model setup allows us to derive an approximate analytical solution for the general equilibrium in the case where the representative agents has CRRA-utility. A key model device is the assumption of the central bank following a Taylor rule, which links the real with the nominal side of the economy and turns out to be crucial in reproducing the salient features of the nominal term structure and its volatility curve.

Our general equilibrium framework builds upon Buraschi \& Jiltsov (2005). However, the key distinction is that for our representative agent we depart from the log-utility assumption and impose a constant relative risk aversion (CRRA) utility. It is well known that for such a utility specification, no closed-form solution for the term structure of interest rates can be obtained. Using perturbation methods, we find that the agents' optimal controls remain affine in the state variables up to a first order approximation in the risk aversion coefficient. This result allows us to study the effect of changing risk aversion on the term structure of interest rates and the yield volatility curve, which previous papers such as Buraschi \& Jiltsov (2005) or Ulrich (2013) were not able to do, and which turn out to be non-trivial.

In our model, both government and monetary policy uncertainty are affecting nominal yields, the term structure of volatility, and bond risk premia in a fundamentally different way. An increase in government policy uncertainty adversely affects the trend component of real output growth. Therefore, it renders capital investments more risky, which will eventually induce investors to favor safe assets such as government bonds. Such a flight-to-quality behavior will raise government bond prices and therefore drives down its yields. This observation is in line with Bloom (2009), who 
argues that productivity growth falls, because higher uncertainty causes firms to temporarily pause their investment. Moreover, in our model economy higher government policy uncertainty will not only negatively affect the long run growth path of production. It also increases its volatility and therefore leads to a worsening of economic growth prospects, which are fundamental to the agents consumption-investment allocation problem. ${ }^{1}$

Not only does government policy uncertainty play an important role in determining the level of interest rates, but it has also a crucial impact on the level and shape of the term structure of bond yield volatilities. Although our model belongs to the class of affine models as introduced by Duffie \& Kan (1996), we can replicate the typical hump-shape of the volatility term structure, caused by the empirical observation that volatility tends to be highest around the two-year maturity bucket. The key mechanism leading to this result is that government policy uncertainty negatively affects the long run growth path of productivity, which translates into a hump-shaped curve at a higher volatility level. With this amplification mechanism we can also explain the 'excess bond yield volatility puzzle' that empirical bond yields, especially at the long end of the term structure, cannot be reproduced by standard affine models of the term structure of interest rates (see Shiller (1979) and Piazzesi \& Schneider (2006)). ${ }^{2}$

Dealing wit policy uncertainty, a fundamental question that arises in this context is: What is an appropriate measure for government and monetary policy uncertainty? As starting point, we use the economic policy uncertainty (EPU) index developed by Baker et al. (2012). According to their definition, the EPU index contains uncertainty related to both government and monetary policy. Hence, we aggregate the corresponding EPU constituents into a government (GPU) and a monetary policy uncertainty (MPU) index.

[Figure 1 about here]

\footnotetext{
${ }^{1}$ Our model is similar in style to the long run risk model of Bansal \& Yaron (2004). However, the key distinctive difference is that the long run growth component and the market price of output risk are both driven by the same underlying risk factor, namely government policy uncertainty. Furthermore, our setting can also be compared to the literature on real business cycle analysis. For instance, shocks to trend growth exhibit fundamentally different effects on the (real) economy as opposed to transitory fluctuations. The agents or county's reaction to temporary shocks is to borrow in the short run to smooth out consumption. However, if the shock is more persistent, the long run consumption level has to be adjusted as borrowing for an infinite time horizon is not longer possible.

${ }^{2} \mathrm{~A}$ possible solution to this problem is to introduce heterogeneous agents who have different prior beliefs about some fundamental economic variable, such as for instance inflation as in Xiong \& Yan (2010) or to introduce time-varying risk preference as in Buraschi \& Jiltsov (2007), which are however analytically less tractable.
} 
In Figure 1 we plot the relationship between U.S. treasury bond yields and volatilities together with the EPU, GPU, and MPU index for the period of 1995 to 2014. The first two prominent spikes of the EPU index are related to the terrorist attacks on the World Trade Center and the 2nd Gulf War. By the end of 2003 and until the outbreak of the financial crisis in 2008, the US economy entered a steady economic growth phase. Both, the EPU and GPU show similar patterns. They declined in the pre-crisis period and started to peak at the onset of the financial crisis. The EPU and GPU index remain at a high level ever since, exhibiting highly volatile behavior. In contrast, the MPU index slowly reversed to its pre-crisis level. We attribute this observation to the fact that the EPU and GPU index captures political uncertainty, which was especially high during the debt-ceiling crisis of 2011 and lasted until late 2013 where some governmental authorities were even forced to suspend their services temporarily.

Figure 1 also shows that both the EPU and GPU index exhibit a countercyclical pattern with nominal yields. When the EPU or GPU index is high, yields tend to go down. This apparent negative relationship is also confirmed by computing the sample correlation coefficient, which ranges from 0.544 (EPU, 1 year) to -0.32 (GPU, 10 years) for the period January 1990 until June 2014. ${ }^{3}$ These numbers suggest that higher economic or government policy uncertainty leads to lower treasury bond yields. Thus, as political risk increases, investors seek safer assets and therefore start to shift from stocks to (government) bonds, which is in line with the predictions in Pastor \& Veronesi (2013). ${ }^{4}$

The discussion above suggests that government and monetary policy uncertainty may have different effects on yields and volatilities. Therefore, we not only split economic uncertainty into these two different components, but we allow government policy uncertainty to play a role for interest rate policy. The empirical analysis of the model confirms our theoretical prediction in that government policy uncertainty is the main driver in contemporaneous movements in the term structure of interest rates and its volatility curve.

\footnotetext{
${ }^{3}$ The time series correlation between the EPU and GPU (MPU) is 0.857 (0.657) and 0.572 between the GPU and MPU index. As can be inferred from Figure 1, the monetary policy uncertainty index appears to have no link with contemporaneous movements in nominal yields. Indeed, the estimated time series correlation is roughly zero along the entire term structure. We collect the all empirical sample correlation of treasury bond yields and the EPU, GPU and MPU indexes in Table 6 as well as realized volatility and EPU, GPU and MPU indexes in Table 7 in Appendix B. Furthermore, the sample correlation between the EPU and the VIX index is 0.44 for the same period.

${ }^{4}$ Using also the EPU index of Baker et al. (2012), they show that political uncertainty raises not only the equity risk premium but also the volatilities and correlations of stock returns.
} 
There is increasing evidence that policy uncertainty leads to direct reactions of the central bank authority (see for instance David \& Veronesi (2014)). To motivate a link between government policy uncertainty and yields for our model design, we estimate pairwise Vector Auto Regressions (VAR) for the GPU and MPU with the three-month T-bill rate, which we take as proxy for monetary policy.

[Figure 2 about here]

Figure 2 reports the resulting impulse responses for the time period from January 1995 to June 2014. Panel A reveals that a shock to the GPU index leads to a sustained negative impact on the short-term rate and hence on future monetary policy. This impact remains highly significant up to a time horizon of more than 20 months. In contrast, from Panel B we observe that a shock to the short-term rate has no significant impact on the GPU index. ${ }^{5}$ This suggests that government policy uncertainty shocks drives monetary policy actions, but not the other way around. Hence, the central bank conducts its monetary policy taking into account uncertainty shocks from the real side whereas the central banks interest rate policy does not seem to affect fiscal uncertainty.

We provide a theoretical explanation for the empirical results above. In our model economy, higher real uncertainty lowers productivity growth, which feeds into the monetary policy through our assumption that the central bank controls the money supply growth following a Taylor rule. Hence, the monetary authority's efforts to stabilize growth (and inflation) causes it to react to real uncertainty by lowering the cost of capital. Moreover, since we assume money neutrality, nominal shocks do not have an impact on the real side of the economy. However, the converse is not true. The equilibrium price level growth is driven by the capital accumulation growth, which implies that the nominal side is also driven by shocks from the real side, namely government policy shocks. Through these two transmission channels, inflation and capital accumulation growth targeting, the money supply growth becomes a function of government policy uncertainty. Therefore, by letting the central bank react endogenously to deviations from long run capital growth and inflation targets establishes an important link between the real and the nominal side of the economy that allows

\footnotetext{
${ }^{5}$ Further analyzing the impulse responses from our VAR model, we find that the MPU index has a similar behavior as the GPU index in that it does (negatively) influence monetary policy, but the MPU does not respond to a shock in the short rate. Furthermore, the response of the short rate from a MPU shock is less pronounced than from a GPU shock. Finally, the MPU and GPU responses to GPU and MPU shocks are negligible and become insignificant after four to five months. We do not report these graphs here, but they can be obtained on request.
} 
government policy shocks to affect nominal quantities. This link proves to be essential in order to simultaneously match both the term structure of interest rates and its corresponding volatility curve.

Finally, we allow both monetary and government policy shocks to carry a risk premium. Hence, our model accommodates a time-varying risk premia in bond returns, which implies that monetary and government policy uncertainty are priced risk factors and the equilibrium inflation process exhibits heteroskedastic time-variation in both variables.

While our paper belongs to the class of equilibrium finance models of the term structure, it is specifically related to the following strands of literature. ${ }^{6}$ Traditional work on asset pricing usually abstracts from modeling government uncertainty and its impact on asset prices. However, especially since the European debt crisis starting in 2010 and the 2011 Congress debate about raising the fiscal debt ceiling in the US, policy uncertainty has recently attracted interest from academia. For instance, Pastor \& Veronesi (2012) and Pastor \& Veronesi (2013) develop a general equilibrium model, in which the profitability of firms is driven by government policy, and discuss the impact of policy risk on stock prices. Several empirical papers have shown that uncertainty about political outcomes has a significant effect on asset returns and corporate decisions. ${ }^{7}$ There is also a large strand of literature trying to infer political risk from government bond yields such as, e.g., Huang et al. (2015) who empirically study the relationship between political risk and government bond yields. For an overview of this literature, we refer to Bekaert et al. (2014).

While the literature on government policy impacts is sparse but growing, the fundamental link between monetary policy and the term structure of interest rates and volatilities has been studies

\footnotetext{
${ }^{6}$ Equilibrium term structure models include, among many others, Wachter (2006), Piazzesi \& Schneider (2006), Buraschi \& Jiltsov (2007), Gallmeyer et al. (2007), Bekaert et al. (2009), and Bansal \& Shaliastovich (2013). Recently, macro-finance term structure models have been critisized for their failure to accomodate for the presence of "unspanned macroeconomic risk," see Joslin, Priebsch \& Singleton (2014). However, there is support for spanned macro-finance term structure models. Basically, Bauer \& Rudebusch (2015) claim to have resolved the unspanning puzzle. Although our model belongs to the spanned model class, we do not delve further into this discussion but refer the interested reader to the two cited papers above.

${ }^{7}$ For instance, early studies include Rodrik (1991) or Pindyck \& Solimano (1993). They show empirically that uncertainty about political factors can lead to lower investment expenditures, especially when investment is irreversible. More recently, Durnev (2010) and Julio \& Yook (2012) document that firms tend to withhold their investment activity prior to national elections. Gulen \& Ion (2012) argue, based on the newly developed policy index by Baker et al. (2012), that policy uncertainty reduces firm and industry level investment and that the magnitude of reduction is substantial. Boutchkova et al. (2012) take the analysis further and show that some industries are more sensitive to political uncertainty than others. Some further related articles analyzing the relationship between political uncertainty and asset returns include Belo et al. (2013), Bialkowski et al. (2008) or Bond \& Goldstein (2012).
} 
more extensively. For the yield effects we refer to, e.g., Kuttner (2001), Piazzesi (2005), Fleming \& Piazzesi (2005), Gürkaynak et al. (2005a), and Wright (2012). For the volatility effects see, e.g., Balduzzi et al. (2001), Piazzesi (2005) or de Goeij \& Marquering (2006), among others. The literature on the link between monetary policy and bond risk premia is surveyed in Buraschi et al. (2014). In an empirical study, they find that monetary shocks are indeed an important source of priced risk, helping to explain the risk premia in bond markets. Using a standard predictability regression of excess bond returns, they find that monetary policy shocks account for a substantial part of the variance of bond excess returns, which is in line with our empirical results.

Despite the recent attention brought to modeling the impact of policy uncertainty on asset prices, the papers mentioned above either address the empirical link between government bond yields and policy uncertainty or focus on the theoretical impact that a given government policy has on stock returns. Our paper provides a theoretical framework for studying the impact of both government and monetary policy uncertainty on the nominal yield curve and its implications for the term structure of bond yield volatility.

The reminder of the paper is organized as follows. Section 2 presents the model. Section 3 discusses the impact of government as well as monetary policy uncertainty on the term structure of nominal interest rates, the yield volatility curve and the bond risk premium. Section 4 summarizes our empirical results and Section 5 concludes.

\section{The baseline model economy}

Our model economy consists of a real and a monetary sector. For the real sector, we consider a production economy with a representative agent producing a single good at a constant return-toscale production technology. As in Buraschi \& Jiltsov (2005), real monetary holdings $M_{t}^{d}$ provide a transaction service by reducing the total amount of gross resources required for a given level of consumption $C_{t}$.

Assumption 1 (Preferences of Representative Agent). Let $\mathcal{U}\left(X_{t}\right)$ denote expected utility over the real net consumption holdings $X$ and $\beta>0$ the subjective discount factor. The agent has constant 
relative risk aversion (CRRA) preferences given by

$$
\mathcal{U}\left(X_{t}\right)=E_{t}\left[\int_{t}^{\infty} e^{-\beta(u-t)} U\left(X_{u}\right) d u\right]
$$

with

$$
U\left(X_{t}\right)= \begin{cases}\frac{1}{\gamma}\left(X_{t}^{\gamma}-1\right), & \text { if } \gamma<1, \gamma \neq 0 \\ \log \left(C_{t}\right)+\xi \log \left(M_{t}^{d}\right) & \text { if } \gamma=0,\end{cases}
$$

where $\gamma$ is equal to one minus the coefficient of risk aversion. In addition, the real net consumption holdings depend on both consumption $C_{t}$ and real cash balances $M_{t}^{d} .8$

$$
X_{t}=C_{t}\left(M_{t}^{d}\right)^{\xi}, 0 \leq \xi \leq 1
$$

The agent's real output is denoted by $Y_{t}$. The drift of $Y_{t}$ is influenced by a productivity factor $A_{t}$ that depends on a process $g_{t}$, which we refer to as government policy or real uncertainty.

Assumption 2 (Real Sector Dynamics). Given a filtered probability space $\left(\Omega, \mathcal{F},\left\{\mathcal{F}_{t}\right\}_{t \geq 0}, \mathbb{P}\right)$ satisfying the usual conditions, the dynamics of real output $Y_{t}$, productivity $A_{t}$, and government policy uncertainty $g_{t}$ have the following dynamics:

$$
\begin{aligned}
& \frac{d Y_{t}}{Y_{t}}=\left(\mu_{Y}+q_{A} A_{t}\right) d t+\sigma_{Y} \sqrt{g_{t}} d W_{t}^{Y}, Y_{0} \in \mathbb{R}_{+}, \\
& d A_{t}=\left(\kappa_{A}\left(\theta_{A}-A_{t}\right)+\lambda g_{t}\right) d t+\sigma_{A} \sqrt{g_{t}} d W_{t}^{A}, \quad A_{0} \in \mathbb{R}, \\
& d g_{t}=\kappa_{g}\left(\theta_{g}-g_{t}\right) d t+\sigma_{g} \sqrt{g_{t}} d W_{t}^{g}, g_{0} \in(0, \infty),
\end{aligned}
$$

with constants $\mu_{Y}>0, q_{A}, \theta_{A}, \lambda, \kappa_{i}>0, \forall i \in\{A, g\}, \sigma_{j}^{2}>0$, and $\mathbb{P}$-Brownian motions $W_{t}^{j}, \forall j \in$ $\{Y, A, g\}$. The processes in (4) to (6) may have nonzero instantaneous correlation:

$$
d W_{t}^{Y} d W_{t}^{A}=\rho^{A Y} d t, \quad d W_{t}^{Y} d W_{t}^{g}=\rho^{Y g} d t, \quad d W_{t}^{A} d W_{t}^{g}=\rho^{A g} d t
$$

Productivity $A_{t}$ in Equation (5) is a mean reverting process with a trend and diffusion term that depend on government policy uncertainty $g_{t}$. Hence, not only is the productivity's long run mean stochastic, but also its volatility is time-varying. A crucial role is played by the parameter $\lambda$. If

\footnotetext{
${ }^{8}$ If $\xi=0$, money does not provide any service and $\xi=1$ implies that the agent needs to hold exactly one unit of currency for every unit of consumption holdings. Since $0 \leq \xi \leq 1$, a higher level of monetary holdings provides a higher level of transaction services, but at a decreasing return to scale.
} 
$\lambda<0(\lambda>0)$, an increasing $g_{t}$ will have a negative (positive) effect on long run productivity. If $\lambda=0$, we obtain a process with constant long run mean $\theta_{A}$ and time-varying volatility.

The government policy uncertainty process $g_{t}$ in Equation (6) describes an unconditionally meanreverting stationary process. It not only affects productivity $A_{t}$, but also the output growth rate $d Y_{t} / Y_{t}$ through two channels. First, $g_{t}$ renders output volatility time-varying which, as we will prove in a later section, leads to a stochastic real market risk. Second, it affects the trend output growth rate indirectly as it influences the growth rate of productivity. For example, if $\lambda<0$, higher government policy uncertainty will reduce the long run level of productivity. Provided that $q_{A}>0$, this reduction leads to a decline in expected output growth. To get some further intuition about our model design, we derive the unconditional expectation, variance, and covariance of productivity and government uncertainty below.

Proposition 1 (Stationary moments of productivity and government policy uncertainty process). The unconditional expectation and variance of productivity $A_{t}$ and government policy uncertainty $g_{t}$, and their covariance are given $b y^{9}$

$$
\begin{aligned}
\mathbb{E}\left[A_{t}\right] & =\lim _{T \rightarrow \infty} \mathbb{E}_{t}\left[A_{T}\right]=\theta_{A}+\frac{\lambda \theta_{g}}{\kappa_{A}}, \\
\mathbb{V}\left[A_{t}\right] & =\lim _{T \rightarrow \infty} \mathbb{V}_{t}\left[A_{T}\right]=\theta_{g}\left(\frac{\sigma_{A}^{2}}{2 \kappa_{A}}+\frac{2 \kappa_{g} \lambda \rho^{A g} \sigma_{A} \sigma_{g}+\lambda^{2} \sigma_{g}^{2}}{2 \kappa_{A} \kappa_{g}\left(\kappa_{A}+\kappa_{g}\right)}\right), \\
\mathbb{E}\left[g_{t}\right] & =\lim _{T \rightarrow \infty} \mathbb{E}_{t}\left[g_{T}\right]=\theta_{g}, \mathbb{V}\left[g_{t}\right]=\lim _{T \rightarrow \infty} \mathbb{V}_{t}\left[g_{T}\right]=\frac{\theta_{g} \sigma_{g}^{2}}{2 \kappa_{g}}, \\
\mathbb{C}\left[A_{t}, g_{t}\right] & =\lim _{T \rightarrow \infty} \mathbb{C}_{t}\left[A_{T}, g_{T}\right]=\frac{\theta_{g} \sigma_{g}\left(2 \kappa_{g} \rho^{A g} \sigma_{A}+\lambda \sigma_{g}\right)}{2 \kappa_{g}\left(\kappa_{A}+\kappa_{g}\right)} .
\end{aligned}
$$

A first important observation is that the long run level of government policy uncertainty $\theta_{g}$ affects all moments. First it raises (lowers) the unconditional expected productivity growth whenever $\lambda>0$ $(\lambda<0)$. Second, it not only raises the stationary long run level of $g_{t}$ proportionally, but also the variance of $A_{t}$ and $g_{t}$. Hence, an increase in $\theta_{g}$ will lead to higher government policy uncertainty and simultaneously also to a higher variance of productivity.

Assuming that the impact of government policy uncertainty on the drift of productivity is negative $(\lambda<0)$ and setting $\rho^{A g}=0$ for simplicity, the effect of $\lambda$ has three important implications. First,

\footnotetext{
${ }^{9}$ By $\mathbb{E}[\cdot], \mathbb{V}[\cdot]$, and $\mathbb{C}[\cdot, \cdot]\left(\mathbb{E}_{t}[\cdot], \mathbb{V}_{t}[\cdot], \mathbb{C}_{t}[\cdot, \cdot]\right)$ we denote the unconditional (conditional on $\mathcal{F}_{t}$ ) expectation, variance, and covariance operators.
} 
it lowers expected growth of productivity proportionally to $\theta_{g} / \kappa_{A}$. Second, it increases volatility of $A_{t}$ linearly. Lastly, it renders $\mathbb{C}\left[A_{t}, g_{t}\right]$ negative, which will be of central importance to capture the stylized facts of bond yield volatility within our model.

We can now proceed with the formulation of the agent's capital budget constraint. Without loss of generality, we use capital depreciation as the only cost component. ${ }^{10}$

Assumption 3 (Capital budget constraint). The real return on capital that can either be allocated to consumption $C_{t} d t$ or cash balances $M_{t}^{d} d t$ or reinvested $d K_{t}$ is given by

$$
C_{t} d t+M_{t}^{d} d t+d K_{t}=K_{t} \frac{d Y_{t}}{Y_{t}}-\delta K_{t} d t
$$

where $K_{t} \frac{d Y_{t}}{Y_{t}}$ is the change in total output and $\delta K_{t} d t$ is the capital depreciation with deprecation rate $\delta \in[0,1]$.

Substituting output growth from Equation (4), and rearranging we obtain the capital accumulation process as

$$
\frac{d K_{t}}{K_{t}}=-\left(\frac{C_{t}}{K_{t}}+\frac{M_{t}^{d}}{K_{t}}\right) d t+\left(\mu_{y}+q_{A} A_{t}-\delta\right) d t+\sigma_{y} \sqrt{g_{t}} d W_{t}^{Y} .
$$

The capital accumulation process is decreasing in the optimal control variables consumption $C_{t}$ and money demand $M_{t}^{d}$, which is intuitive as higher $C_{t}$ and/or $M_{t}^{d}$ diminish available resources to be invested in the production technology $K_{t}$. Similar to real output, capital is nonstationary whenever $\mu_{y} \neq \delta$ and time-varying in productivity $A_{t}$. Furthermore, Equation (13) implies money-neutrality, i.e., monetary shocks do not have an effect on the real side of the economy. ${ }^{11}$

\footnotetext{
${ }^{10}$ In our model, we do not include a variable cost component as was done, e.g., in Buraschi \& Jiltsov (2005).

${ }^{11}$ Whether or not real output and capital are money-shock-neutral is debated in macroeconomics for a long time. The neo-classical Keynesian literature argues that any increase in money supply has to be offset by an equivalent proportional rise in prices and wages. A recent paper using a similar setup as ours is Ulrich (2013), who sticks to this neo-classical view. However, there are a number of reasons why inflation may affect the real economy. See, e.g., Fisher \& Modigliani (1978), who argue that inflation has a direct influence on purchasing power, because many private contracts are not indexed. A first quantitative study that allows for dependence of the expected return on capital on inflation is Pennacchi (1991), who uses survey data to identify inflationary expectations. Another channel through which inflation can affect the real economy is through taxation of nominal asset returns. This channel was exploited by Buraschi \& Jiltsov (2005) to account for the violation of the expectation hypothesis and the determination of the inflation risk premium. Since policy uncertainty affects the real and nominal side of the economy (through the endogenous equilibrium price level), we assume money-neutrality throughout the paper. However, we acknowledge that a feasible extension of our model is to let the capital accumulation process be a function of the price level. We leave this as an interesting theoretical idea which is worthwhile to be considered.
} 
For the monetary sector, we assume that there exists a central bank controlling money supply $M_{t}^{S}$ on the basis of a Taylor rule. The monetary authority targets a long term nominal constant money growth rate $\mu_{M}$, a capital growth rate $\bar{k}$, and an inflation rate equal to $\bar{\pi}$. We assume that transitory deviations from the optimal long run money growth exhibit stochastic volatility. We can interpret this time-variation in money supply, denoted by $m_{t}$, as the monetary policy uncertainty factor.

Assumption 4 (Monetary Sector). The central bank controls money supply growth according to a Taylor rule as follows:

$$
\begin{aligned}
\frac{d M_{t}^{S}}{M_{t}^{S}} & =\mu_{M} d t+\eta_{1}\left(\frac{d K_{t}}{K_{t}}-\bar{k} d t\right)+\eta_{2}\left(\frac{d p_{t}}{p_{t}}-\bar{\pi} d t\right)+\sigma_{M} \sqrt{m_{t}} d W_{t}^{M} \\
d m_{t} & =\kappa_{m}\left(\theta_{m}-m_{t}\right) d t+\sigma_{m} \sqrt{m_{t}} d W_{t}^{m}, \quad d W_{t}^{M} d W_{t}^{m}=\rho^{M m} d t
\end{aligned}
$$

where $p_{t}$ and $K_{t}$ are the price level and capital accumulation process. We assume that the monetary policy innovation correlates with the money supply by $\rho^{M m}$, but is independent of all other sources of risk in the economy.

The crucial parameters in Assumption 4 are $\eta_{1}$ and $\eta_{2}$ in Equation (14). Their magnitude determines the sensitivity of money supply growth with respect to deviations of endogenous capital and inflation from their long run target levels. For example, if output growth is above target $\left(\frac{d K_{t}^{*}}{K_{t}^{*}}-\right.$ $\bar{k} d t>0)$ and provided that $\eta_{1}<0$, the monetary authority shrinks the money supply, causing interest rates to rise and investment activity to slow down. When inflation is below target $\left(\frac{d p_{t}^{*}}{p_{t}^{*}}-\bar{\pi} d t<0\right)$ and provided that $\eta_{2}<0$, the central bank's response is to increase the money supply. In the case when $\eta_{1}=\eta_{2}=0$ money supply is exogenous and therefore does not react to deviations from long run capital nor inflation growth rate. Furthermore, given the money supply rule in Equation (14), monetary policy uncertainty renders money supply time-varying in $m_{t}$. Having introduced both the real and monetary side of the economy, we next characterize the representative agent's equilibrium.

Definition 2 (Equilibrium Capital Stock and Money Holdings). Under Assumptions 1 to 4, the representative agent's equilibrium is defined as a vector of optimal consumption, money demand, price and capital processes $\left[C_{t}^{*}, M_{t}^{d *}, K_{t}^{*}, p_{t}^{*}\right]$ that is a solution to the following dynamic Hamilton- 
Jacobi-Bellmann programming problem ${ }^{12}$

$$
0=\frac{\partial V\left(t, K_{t}, A_{t}, g_{t}\right)}{\partial t}+\max _{\left\{C_{t}, M_{t}^{d}\right\}}\left\{U\left(C_{t}, M_{t}^{d}\right)+\mathcal{A} V\left(t, K_{t}, A_{t}, g_{t}\right)\right\},
$$

subject to money market-clearing $M_{t}^{S}=p_{t}^{*} M_{t}^{d *}$, the bugdet constraint in (12), and the transversality condition $\lim _{T \rightarrow \infty} \mathbb{E}_{t}\left[e^{-\beta T} V\left(t, K_{t}, A_{t}, g_{t}\right)\right]=0$.

For the problem in Equation (16), an explicit solution can only be obtained for the log-utility case. The resulting optimal consumption and money demand holdings are proportional to capital $K_{t}$. However, for $\gamma \neq 0$, the asymptotic optimal controls $C_{t}$ and $M_{t}^{d}$ remain linear in the state variables $K_{t}, A_{t}$, and $g_{t}$ (up to a first-order approximation in $\gamma$ ). This feature allows us to find an explicit affine representation of the term structure of real and nominal interest rates beyond the log-utility case. $^{13}$

Proposition 3 (Perturbed equilibrium of the representative agent's investment and consumption problem). In equilibrium, the representative agent's value function is

$$
V\left(t, K_{t}, A_{t}, g_{t}\right)=\frac{e^{-\beta t}}{\beta \gamma}\left(\left(e^{\phi\left(A_{t}, g_{t}\right)} K_{t}^{1+\xi}\right)^{\gamma}-1\right)
$$

for some function $\phi\left(A_{t}, g_{t}\right)$ of the form

$$
\phi\left(A_{t}, g_{t}\right)=\phi_{0}\left(A_{t}, g_{t}\right)+\gamma \phi_{1}\left(A_{t}, g_{t}\right)+O\left(\gamma^{2}\right)
$$

The agent's first order asymptotic optimal controls are

$$
C_{t}^{*}=\frac{\beta K_{t}}{1+\xi}\left[1+\gamma\left(L-\phi_{0}\left(A_{t}, g_{t}\right)\right)\right], \quad M_{t}^{d *}=\xi C_{t}^{*}
$$

and the equilibrium first order asymptotic capital accumulation $K_{t}^{*}$ and price process $p_{t}^{*}$ satisfy

$$
\begin{aligned}
\frac{d K_{t}^{*}}{K_{t}^{*}} & =\mu_{K^{*}}\left(A_{t}, g_{t}\right) d t+\sigma_{Y} \sqrt{g_{t}} d W_{t}^{Y} \\
\frac{d p_{t}^{*}}{p_{t}^{*}} & =\left[\frac{\mu_{M}-\eta_{1} \bar{k}-\eta_{2} \bar{\pi}}{1-\eta_{2}}+\frac{\eta_{1}-1}{1-\eta_{2}} \mu_{K^{*}}\left(A_{t}, g_{t}\right)-g_{t} \frac{\left(\eta_{1}-1\right) \sigma_{Y}^{2}}{1-\eta_{2}}\right] d t \\
& +\frac{\sigma_{M} \sqrt{m_{t}}}{1-\eta_{2}} d W_{t}^{M}+\frac{\left(\eta_{1}-1\right) \sigma_{Y} \sqrt{g_{t}}}{1-\eta_{2}} d W_{t}^{Y} .
\end{aligned}
$$

\footnotetext{
${ }^{12}$ By $\mathcal{A}$ we denote the infinitesimal generator. See, e.g., Øksendal (2003) for technical details.

${ }^{13}$ Our solution strategy is based on the perturbation method. In particular, we follow the approach of Kogan \& Uppal (2001) and approximate our model with respect to the risk aversion parameter around the explicit equilibrium computed under the log-utility assumption. Perturbation methods have been successfully applied in many other studies such as, e.g., Hansen et al. (2008).
} 
where

$$
\mu_{K^{*}}\left(A_{t}, g_{t}\right):=\mu_{Y}+q_{A} A_{t}-\beta-\delta+\gamma \beta\left(\phi_{0}\left(A_{t}, g_{t}\right)-L\right)
$$

denotes the equilibrium drift of the capital accumulation process and $L=\log \left(\frac{\beta^{1+\xi} \xi \xi}{(1+\xi)^{1+\xi}}\right)$ is a constant. Furthermore,

$$
\phi_{0}\left(A_{t}, g_{t}\right)=\phi_{00}+\phi_{0 A} A_{t}+\phi_{0 g} g_{t}
$$

is an affine function in the state variables $\left(A_{t}, g_{t}\right)$ with constants $\phi_{00}, \phi_{0 A}, \phi_{0 g}$ provided in Appendix A.2.

Since nominal shocks have no real effects, the equilibrium capital accumulation process is only driven by the real sector of the economy, i.e., by productivity $A_{t}$ and by the government policy uncertainty process $g_{t}$. Note that for $\gamma=0$ the equilibrium capital drift $\mu_{K^{*}}$ becomes independent of $g_{t}$. The weighting factor $\eta_{2}$ on inflation-target deviation enters non-linearly into the equilibrium price process. ${ }^{14}$

Proposition 3 also implies that the equilibrium price process is driven by both real and monetary shocks. This result is a consequence of the central bank authority controlling money supply growth based on a Taylor-rule. Hence, by endogenizing money supply growth, we allow for an important link between the real and the nominal sector and government policy uncertainty enters the nominal side of the economy through two different channels, namely though its impact on both the equilibrium capital accumulation process and inflation. This link will prove to be essential to capture key empirical properties of the yield curve and its corresponding term structure of yield volatility.

\section{The term structure of nominal interest rates}

Having obtained the dynamics of the equilibrium price level, we can now solve for the term structure of nominal and real bond prices. Let $B(t, \tau)$ be the nominal pure discount bond paying one unit of

\footnotetext{
${ }^{14}$ Note that for $\eta_{2} \approx 1$, small innovations in either $A_{t}, g_{t}$ or $m_{t}$ result in dramatic changes in the equilibrium price process. However, from an economic viewpoint, the parameter $\eta_{2}$ should be negative which, as we will see later, is confirmed by the data.
} 
currency in $t+\tau$ periods. The price of the nominal bond must satisfy the following Euler equation

$$
B(t, \tau)=e^{-\beta \tau} \mathbb{E}_{t}\left[\frac{U_{C}\left(C_{t+\tau}^{*}, M_{t+\tau}^{d *}\right)}{U_{C}\left(C_{t}^{*}, M_{t}^{d *}\right)} \frac{p_{t}^{*}}{p_{t+\tau}^{*}}\right]=e^{-\beta \tau} \mathbb{E}_{t}\left[\frac{\exp \left\{-\log \left(K_{t+\tau}^{*}\right)\right\}}{\exp \left\{-\log \left(K_{t}^{*}\right)\right\}} \frac{p_{t}^{*}}{p_{t+\tau}^{*}}\right],
$$

which states that in equilibrium the investor should be indifferent between consuming one more unit of currency now or investing one unit of currency in the $t+\tau$ period nominal discount bond.

Proposition 4 (Equilibrium Nominal Term Structure of Interest Rates). Under time-separable CRRA utility as in Equation (2), the nominal discount bond $B(t, \tau)$ with maturity $\tau$ is given by

$$
B(t, \tau)=\exp \left\{-b_{0}(\tau)-b_{A}(\tau) A_{t}-b_{g}(\tau) g_{t}-b_{m}(\tau) m_{t}\right\}
$$

where

$$
\begin{aligned}
b_{A}(\tau) & =C_{A} \frac{1-e^{-\kappa_{A} \tau}}{\kappa_{A}} \\
-b_{g}^{\prime}(\tau) & =Z_{0 g}(\tau)+Z_{1 g}(\tau) b_{g}(\tau)+Z_{2 g} b_{g}^{2}(\tau) \\
b_{m}(\tau) & =\frac{-Z_{1 m}+H_{m} \operatorname{Cot}\left(\frac{1}{2}\left(-H_{m} \tau-\operatorname{Tan}\left(\frac{2 \sqrt{Z_{0 m} Z_{2 m}}}{H_{m}}\right)\right)\right)}{2 Z_{2 m}}, H_{m}=4 Z_{0 m} Z_{2 m}-Z_{1 m}^{2}, \\
b_{0}(\tau) & =\int_{0}^{\tau} C_{0}(u) d u
\end{aligned}
$$

and the constant parameters $Z_{0 m}, Z_{2 i}, i \in\{g, m\}$ and $Z_{0 g}(\tau), Z_{1 g}(\tau), C_{0}(\tau)$ are time-to-maturity functions that only depend on the structural model parameters of the economy and are defined in Appendix A.3.

The nominal term structure of interest rates in Proposition 4 belongs to the general affine class of term structure models of discount bond prices introduced by Duffie \& Kan (1996). Using Equation (25), we obtain the time $t$ yield curve $Y(t, \tau)$ with maturity $\tau$ as

$$
Y(t, \tau):=-\frac{1}{\tau} \log (B(t, \tau))=\frac{b_{0}(\tau)}{\tau}+\frac{b_{A}(\tau)}{\tau} A_{t}+\frac{b_{g}(\tau)}{\tau} g_{t}+\frac{b_{m}(\tau)}{\tau} m_{t}
$$

The affine yield model in Equation (30) is driven by three factors. As noted, e.g., by Litterman \& Scheinkman (1991), such a three factor structure model of the term structure is able to reproduce most if not all empirically relevant shapes of the yield curve, such as upward sloping, inverted or hump shapes. The affine property in the factor loadings implies linearity of the local variance as in Vasicek (1977), Cox et al. (1985), and others. 
Analyzing the expressions for the constant and time-varying parameters $Z_{\text {.,., }} Z_{0 g}(\tau)$, and $C_{0}(\tau)$ given in Appendix A.3, we make the following three observations. First, the target growth rates for output $\bar{k}$ and inflation $\bar{\pi}$, the depreciation rate $\delta$, and output $\mu_{Y}$ and money supply growth rate $\mu_{M}$ solely affect the intercept of the yield curve but not its slope. In contrast, their weighting factors $\eta_{1}$ and $\eta_{2}$ affect both the intercept and the slope of the yield curve. Moreover, they do so in a non-linear way.

Second, the parameter $\lambda$ has a key impact not only on the level of the term structure but also on its slope. The parameter $\lambda$ affects the level of yields, since both $C_{A}=C_{A}(\lambda)$ and $C_{g}=C_{g}(\lambda)$ enter the expression for $b_{0}(\tau)$ and are functions of $\lambda$. This dependence in turn implies that $\lambda$ also affects the slope of the term structure through $b_{A}(\tau)$ and $b_{g}(\tau)$. Furthermore, $\lambda$ also determines the long run level of productivity $A_{t}$. To see this, recall that the trend growth rate of the productivity process $A_{t}$ is not only dependent on the long run level of productivity $\theta_{A}$ but also on the long run

level government policy $\theta_{g} \cdot{ }^{15}$ Suppose that $\theta_{g}>0$ and $\lambda<0$, and $\theta_{A}+\frac{\lambda \theta_{g}}{\kappa_{A}}<0$, the term $\frac{b_{A}(\tau)}{\tau} A_{t}$, provided that $b_{A}(\tau)>0$, will be positive with high probability and therefore will lead to a declining yield curve for any maturity.

Third, the subjective discount factor $\beta$ and the degree of transaction service money provides $\xi$ also impact the slope of the yield curve through the factor loadings $b_{A}(\tau)$ and $b_{g}(\tau)$ whenever $\gamma \neq 0$. Hence, if the representative agent would have $\log$-utility, $\beta$ and $\xi$ would only exhibit a level effect. In the following section we explore the impact of government and monetary policy uncertainty on the nominal term structure of interest rates in more detail.

\section{1 $\quad$ Fitting the model to the data}

To fit the model to the term structure data we proceed as follows. We estimate a subset of parameters using maximum likelihood estimation (MLE) and calibrate the remaining parameters to the nominal yield and its corresponding volatility curve simultaneously. We estimate the parameters of the policy uncertainty variables $g_{t}$ and $m_{t}$ using (exact) maximum likelihood estimation. ${ }^{16}$ Table 1 summarizes

\footnotetext{
${ }^{15}$ In particular, we have $\mathbb{E}\left[A_{t}\right]=\theta_{A}+\frac{\lambda \theta_{g}}{\kappa_{A}}$ (see Equation (8) in Proposition 1).

${ }^{16}$ Since for the Feller diffusion the transition density is known in closed-form (non-central chi-squared), the transition density does not need to be approximated via quasi maximum likelihood techniques.
} 
the results.

\begin{tabular}{lcccccc}
\hline & \multicolumn{3}{c}{ GPU } & \multicolumn{3}{c}{ MPU } \\
\hline & $\kappa_{g}$ & $\theta_{g}$ & $\sigma_{g}$ & $\kappa_{m}$ & $\theta_{m}$ & $\sigma_{m}$ \\
\hline Estimate & 0.203 & 0.931 & 0.326 & 0.418 & 0.935 & 0.285 \\
Stand. Error & $(0.05)$ & $(0.104)$ & $(0.021)$ & $(0.062)$ & $(0.043)$ & $(0.022)$ \\
\hline
\end{tabular}

Table 1: The label 'GPU' and 'MPU' refer to the government and monetary policy index, respectively. The row 'Estimates' represents the maximum likelihood estimator and the row 'Stand. Error' shows the asymptotic robust standard errors ('Sandwich estimator') of the parameters which is based on the outer product of the Jacobian of the log-likelihood function. Estimation period is January 1990 to June 2014 (295 data points) using monthly data.

The estimated parameters between the GPU index and the MPU index differ mainly in the magnitude of the speed of mean reversion parameter $\kappa$. Table 1 shows that $\kappa_{m}$ is about half the magnitude of $\kappa_{m}$. Hence, a government policy shock will have a more permanent effect than a monetary shock has. ${ }^{17}$ Shocks to monetary policy uncertainty are more transitive and mean-reverts faster to its long run mean $\theta_{m}$.

Next, the parameters related to GDP growth $d Y_{t} / Y_{t}$ and money supply growth $d M_{t}^{S} / M_{t}^{S}$ are set equal to their unconditional first and second sample moments. Furthermore, the correlation parameters $\rho^{Y g}$ and $\rho^{M m}$ are set to their unconditional sample correlation coefficients. The preference parameters $(\beta, \gamma, \xi)$, the structural model parameters $\left(\delta, \eta_{1}, \eta_{2}, \bar{k}, \bar{\pi}\right)$, the correlation parameters $\left(\rho^{A g}, \rho^{A Y}\right)$, and the parameters related to the productivity process $\left(\kappa_{A}, \theta_{A}, \sigma_{A}, q_{A}, \lambda\right)$ are calibrated to match key features of the empirical term structure of interest rates and its corresponding volatility curve. The parameters not related to $g_{t}$ and $m_{t}$ are summarized in Table 2 below.

Given the parameters in Table 1 and 2, we simulate the economy $M=1^{\prime} 000$-times on a monthly basis with each time series having length $12 \times N$, where we set $N=2^{\prime} 500$, using a standard EulerMaruyama scheme to obtain simulated values for the three Itô diffusions $A_{t}, g_{t}$, and $m_{t}$. Having obtained simulated values for those state variables, we then compute the nominal yield curve and its corresponding term structure of bond yield volatility with time to maturity of ten years and then average over the number of Monte-Carlo simulation runs. ${ }^{18}$

\footnotetext{
${ }^{17}$ The half-life of a shock in $g_{t}$ when $\kappa_{g}=0.203$ is $-\log (0.5) / \kappa_{g}=1.48$ months, which implies that it takes a about six weeks for a shock to government policy uncertainty to die out by half. Similarly for the monetary policy shock, we have $-\log (0.5) / \kappa_{m}=0.72$ months. It takes about three weeks for a monetary policy shock to die out by half.

${ }^{18} \mathrm{We}$ set the starting values of the government and monetary policy uncertainty to one $\left(m_{0}=g_{0}=1\right)$. By using a
} 


\begin{tabular}{rrrlrrrr}
\hline \multicolumn{8}{c}{ Model Parameters } \\
\hline$\beta$ & 0.02 & $q_{A}$ & 0.28 & $\sigma_{M}$ & 0.45 & $\kappa_{A}$ & 1.08 \\
$\xi$ & 0.85 & $\bar{k}$ & 0.03 & $\rho^{A Y}$ & 0.14 & $\theta_{A}$ & 4.19 \\
$\gamma$ & -0.82 & $\bar{\pi}$ & 0.03 & $\rho^{A g}$ & -0.98 & $\sigma_{A}$ & 0.27 \\
$\delta$ & 0.08 & $\mu_{Y}$ & 0.38 & $\rho^{g Y}$ & -0.27 & $\lambda$ & -1.93 \\
$\eta_{1}$ & -1.80 & $\sigma_{Y}$ & 0.23 & $\rho^{M m}$ & 0.12 & $A_{0}$ & 1 \\
$\eta_{2}$ & -2.34 & $\mu_{M}$ & 0.26 & & & & \\
\hline
\end{tabular}

Table 2: Summary of parameter values selected for Monte-Carlo Analysis: All parameter values for the process $A_{t}$ as given in Equation (5) and the model parameters $\beta, \gamma, \xi, q_{A}, \delta, \eta_{1}, \eta_{2}, \bar{k}, \bar{\pi}, \rho^{A g}$ and $\rho^{A Y}$ are calibrated to match simultaneously, the average yield curve and bond volatility curve over the sample period January 1990 to June 2014. $A_{0}$ refers to the initial value of $A_{t}$.

[Figure 3 about here]

From Figure 3, Panel A, we see that the model is overestimating the yield curve at medium maturities (three to seven years), whereas at the short and at the long end, the model implied yield curve is underestimating the actual the term structure. However, the overall mean error along the entire term structure is $3.07 \%{ }^{19}$ As Panel B of Figure 3 shows, our fitted model is able to reproduce the hump-shape in the term structure of bond yield volatility. This stylized fact of interest rates is very challenging to replicate within the framework of affine term structure models. Indeed, even with their more flexible specification of essentially affine price of risk, Buraschi \& Jiltsov (2005) acknowledge that their model cannot fully match the second moments of yields. In contrast, we capture the short end of the yield volatility curve very accurately. We slightly overestimate the actual bond volatility at medium to longer maturities. Nevertheless, the error remains with $4.71 \%$ relatively small.

\subsection{Yield curve and policy uncertainty}

We now test a series of implications regarding the effect of policy uncertainty on the yield curve based on our fitted model parameters. Our preliminary empirical analysis between nominal bond yields and policy uncertainty as measured by the EPU index of Baker et al. (2012) shows that there

long time series, we avoid dependence on starting values.

${ }^{19}$ The error is calculated as $\frac{1}{T} \sum_{\tau \in T}|\hat{Y}(t, \tau)-Y(t, \tau)| / \hat{m}$ where $\hat{m}=\frac{1}{T} \sum_{\tau \in T} Y(t, \tau), \hat{Y}(t, \tau)$ is the fitted yield curve and $T=6$, i.e. the number of maturities. 
is significant negative correlation between economic policy uncertainty and movements in the yield curve. Splitting the index into government and monetary policy uncertainty shows that the GPU index maintains high negative correlation whereas the MPU index seems to have no correlation with nominal yields at any maturity. Using our affine yield model, we can compute the model-implied correlation in a straightforward way. More specifically, we can show that a higher level $\left(g_{t}\right.$ or $\left.m_{t}\right)$ in either fiscal or monetary policy uncertainty will lead to a downward shift in yields.

Proposition 5 (Model-implied correlation and impact of policy uncertainty on the yield curve). 1. Nominal yields are negatively correlated with either fiscal $\left(g_{t}\right)$ or monetary $\left(m_{t}\right)$ policy uncertainty, i.e.

$$
\varrho\left[Y(t, \tau), g_{t}\right] \leq 0, \quad \varrho\left[Y(t, \tau), m_{t}\right] \leq 0, \quad \forall \tau \geq 0
$$

and the effect is stronger for fiscal as opposed to monetary policy uncertainty, in other words

$$
\left|\varrho\left[Y(t, \tau), g_{t}\right]\right|>\left|\varrho\left[Y(t, \tau), m_{t}\right]\right|
$$

2. Nominal yields yields are decreasing in both fiscal $\left(g_{t}\right)$ and monetary $\left(m_{t}\right)$ policy uncertainty

$$
\frac{\partial Y(t, \tau)}{\partial g_{t}}=\frac{b_{g}(\tau)}{\tau}<0, \quad \frac{\partial Y(t, \tau)}{\partial m_{t}}=\frac{b_{m}(\tau)}{\tau}<0, \quad \forall \tau \geq 0 .
$$

and this effect is again stronger for fiscal as opposed to monetary policy uncertainty, i.e.,

$$
\left|\frac{b_{g}(\tau)}{\tau}\right|>\left|\frac{b_{m}(\tau)}{\tau}\right|, \quad \forall \tau \geq 0
$$

The results in Proposition 5 above are in line with the empirical observations. The reason why the model implied correlation is negative for any maturity can be directly deduced from the covariance between $Y(t, \tau)$ and $g_{t}$ which is,

$$
\mathbb{C}\left[Y(t, \tau), g_{t}\right]=\frac{b_{A}(\tau)}{\tau} \frac{\theta_{g} \sigma_{g}\left(2 \kappa_{g} \lambda \rho^{A g} \sigma_{A}+\lambda \sigma_{g}\right)}{2 \kappa_{g}\left(\kappa_{A}+\kappa_{g}\right)}+\frac{b_{g}(\tau)}{\tau} \frac{\theta_{g} \sigma_{g}^{2}}{2 \kappa_{g}} .
$$

Given the fitted parameters in Table 1 and 2, the factor loading $b_{A}(\tau)$ is always positive whereas $b_{g}(\tau)$ is negative, which implies that the first and second term in Equation (33) will be negative. In this setting, we obtain a model-implied average (along maturity $\tau$ ) correlation of -0.2934 which is comparable to the empirical sample correlation between nominal yields and the GPU (-0.40). Likewise, since $b_{m}(\tau) \leq 0$ we obtain $\mathbb{C}\left(Y(t, \tau), m_{t}\right)=\frac{\theta_{m} \sigma_{m}^{2}}{2 \kappa_{m}} \frac{b_{m}(\tau)}{\tau} \leq 0, \forall \tau \geq 0$. Comparing this model 
correlation coefficient of -0.021 to its empirical counterpart $(-0.011)$, we see that the model is able to match both sample correlation coefficients.

\subsection{Equilibrium nominal short rate and bond excess returns}

We now discuss how the short end of the term structure of interest rates and the bond risk premium are affected by government and monetary policy uncertainty.

Proposition 6 (Equilibrium nominal short rate and bond risk premium). With time-separable CRRA utility, we have the following first order asymptotic results:

1. The nominal short rate $R_{t}$ is given by

$$
\begin{aligned}
R_{t} & =\frac{\left(\mu_{Y}-\beta-\delta-\bar{k}\right) \eta_{1}+\beta+\mu_{M}-\eta_{2}\left(\mu_{Y}+\bar{\pi}-\delta\right)}{1-\eta_{2}}+\gamma \frac{\beta\left(\eta_{1}-\eta_{2}\right)\left(L-\phi_{00}\right)}{1-\eta_{2}}-\frac{\sigma_{M}^{2}}{\left(\eta_{2}-1\right)^{2}} m_{t} \\
& -\left(q_{A}+\gamma \beta \phi_{0 A}\right)\left(\frac{\eta_{1}-\eta_{2}}{\eta_{2}-1}\right) A_{t}-\left(\frac{\left(\eta_{1}-\eta_{2}\right)^{2} \sigma_{Y}^{2}}{\left(\eta_{2}-1\right)^{2}}+\gamma \frac{\beta \phi_{0 g}\left(\eta_{1}-\eta_{2}\right)}{\eta_{2}-1}\right) g_{t}
\end{aligned}
$$

2. The nominal price of fiscal risk $\lambda_{t}^{N, g}$ as well as the market price of monetary risk $\lambda_{t}^{N, m}$ are

$$
\lambda_{t}^{N, g}=\frac{\eta_{2}-\eta_{1}}{\eta_{2}-1} \sigma_{Y} \sqrt{g_{t}}, \quad \lambda_{t}^{N, m}=\frac{\sigma_{M}}{\eta_{2}-1} \sqrt{m_{t}}
$$

3. The bond risk premia $R P(t, \tau)$ per unit of time is given by

$$
\begin{aligned}
R P(t, \tau) & :=\frac{1}{d t} \mathbb{E}_{t}\left[\frac{d B(t, \tau)}{B(t, \tau)}-R_{t} d t\right] \\
& =\lambda_{t}^{N, Y}\left[b_{A}(\tau) \rho^{A Y} \sigma_{A}+b_{g}(\tau) \rho^{g Y} \sigma_{g}\right] \sqrt{g_{t}}+\lambda_{t}^{N, M} b_{m}(\tau) \rho^{M m} \sigma_{m} \sqrt{m}_{t} \\
& =\Lambda_{t}^{N, g}+\Lambda_{t}^{N, m}
\end{aligned}
$$

where $\Lambda_{t}^{N, g}=\lambda_{t}^{N, Y}\left[b_{A}(\tau) \rho^{A Y} \sigma_{A}+b_{g}(\tau) \rho^{g Y} \sigma_{g}\right] \sqrt{g_{t}}$ and $\Lambda_{t}^{N, m}=\lambda_{t}^{N, M} b_{m}(\tau) \rho^{M m} \sigma_{m} \sqrt{m}_{t}$ are the factor loadings of government and monetary policy uncertainty, respectively.

The nominal short rate and the nominal market price of risk are all influenced by both the real and nominal sector of the economy, which is a direct consequence of the Taylor rule in Assumption 4. In the special case when money supply is entirely decoupled from the real sector $\left(\eta_{1}=\eta_{2}=0\right)$, the nominal short rate reduces to $R_{t}=\mu_{M}+\beta-\sigma_{M}^{2} m_{t}$ and $\lambda_{t}^{N, Y}=0$ so that the real side does 
not affect the nominal short rate and output risk is no longer a nominal risk factor. We get the same result when $\eta_{1}=\eta_{2}=\eta$, i.e., when the central bank reacts to deviations from its nominal and real targets equally. Then, the nominal short rate is also an affine function of $m_{t}$ and the risk premium does not depend on $g_{t}$. In both cases, the nominal short rate becomes independent of the risk aversion parameter $\gamma$.

In the general case, $R_{t}$ depends on the money supply control variables $\eta_{1}$ and $\eta_{2}$ in a non-linear way. This suggests that relatively small fluctuations in either productivity $A_{t}$, monetary or government policy uncertainty may lead to drastic movements in the nominal short rate. Furthermore, risk aversion affects $R_{t}$ through different channels. First, it affects its level through the second term in Equation (34). If risk aversion increases, i.e., if $\gamma$ decreases, the short rate becomes smaller since $\frac{\beta\left(\eta_{1}-\eta_{2}\right)\left(L-\phi_{00}\right)}{\eta_{2}-1}>0$. Secondly, the risk aversion coefficient $\gamma$ loads on both real sector variables $A_{t}$ and $g_{t}$. Therefore, risk aversion also impacts the slope and curvature of the term structure. To what extent risk aversion changes slope and curvature depends on the magnitude of the estimated parameter values.

When $\eta_{1} \neq 0$ and $\eta_{2} \neq 0$, the nominal price of risk decomposes into two state-dependent market prices of risks $\lambda_{t}^{N, Y}$ and $\lambda_{t}^{N, M}$, which are driven by government and monetary policy risk, respectively. ${ }^{20}$ Furthermore, the sign of those market prices of risk is determined by $\eta_{1}$ and $\eta_{2}$, the parameters controlling the intensity of adjustments to the long run real output growth target $\bar{k}$ and inflation target $\bar{\pi}$ and therefore can become negative depending on the values of $\eta_{1}$ and $\eta_{2}$.

[Figure 4 about here]

For the bond risk premium in Equation (37), we plot in Figure 4, Panel A, the loadings of the government policy uncertainty factor $g_{t}$ and the monetary policy uncertainty factor $m_{t}$. Under our estimated parameters, both government and monetary policy uncertainty load positively on the risk premium. Monetary policy uncertainty has a larger impact at the short end of the curve, but flattens out at longer maturities and eventually becomes dominated by government policy uncertainty. Hence,

\footnotetext{
${ }^{20}$ The results in Proposition 6 reveal that equilibrium relations such as the expected bond excess premium and interest rate volatility as well as the forward term premium, i.e., violation of the expectation hypothesis, will be driven by government and monetary policy uncertainty whenever $\eta_{1} \neq 0$ and $\eta_{2} \neq 0$.
} 
our model predicts that the dominant factor at the short end is monetary policy uncertainty, while the dominant role at the long end of the bond risk premia curve is played by the government policy uncertainty factor.

\subsection{Bond yield volatility}

Many empirical studies find that long run bond yields exhibit higher volatility than implied by the expectation hypothesis. Already Shiller (1979) shows that long term bond yields exhibit excess volatility relative to their model-implied values. From Piazzesi \& Schneider (2006) we know that their representative agent-based model explains a smaller fraction of observed volatility of the long-end yields than of the short-end yields. Xiong \& Yan (2010) argue that excess bond volatility might be due to differences in beliefs about the long run level of inflation. They show that a higher belief dispersion leads to volatility amplification which allows them to account not only for the empirically observed high bond yield volatility, but also for the hump-shape of the term structure of bond volatility. ${ }^{21}$ To explore the key determinants that allow to reproduce the hump-shape in bond volatility, we need to derive the model-implied unconditional variance of nominal yields. It turns out that the variance is a linear combination of the variances of monetary and government policy uncertainty, the variance of productivity, and the covariance of productivity and government policy uncertainty.

Corollary 1 (Term Structure of Nominal Bond Yield Variance). Let $Y(t, \tau)$ denote the current time $t$ yield with maturity $\tau$. Then the unconditional term structure of bond yield variance is given by

$$
\mathbb{V}[Y(t, \tau)]=\frac{b_{A}^{2}(\tau)}{\tau^{2}} \mathbb{V}\left[A_{t}\right]+\frac{b_{g}^{2}(\tau)}{\tau^{2}} \mathbb{V}\left[g_{t}\right]+\frac{b_{m}^{2}(\tau)}{\tau^{2}} \mathbb{V}\left[m_{t}\right]+2 \frac{b_{A}(\tau) b_{g}(\tau)}{\tau^{2}} \mathbb{C}\left[A_{t}, g_{t}\right]
$$

where $\mathbb{V}\left[m_{t}\right]=\frac{\theta_{m} \sigma_{m}^{2}}{2 \kappa_{m}}$ and the expressions for $\mathbb{V}\left[A_{t}\right], \mathbb{C}\left[A_{t}, g_{t}\right]$, and $\mathbb{V}\left[g_{t}\right]$ are given in Proposition 1 .

To explore the key determinants in generating the hump-shape in bond yield variance, Figure 4 plots the different components contributing to the bond yield variance in Equation (38).

[Figure 4 about here]

\footnotetext{
${ }^{21}$ Closely related to the 'excess volatility puzzle' phenomenon are also the findings of Gürkaynak et al. (2005b). They document that bond yields exhibit excess sensitivity to macroeconomic announcements.
} 
The contribution of both the factor loadings $b_{g}(\tau)$ and the cross term $b_{A}(\tau) b_{g}(\tau)$ are hump shaped, which causes the term structure of yield variance to exhibit a similar pattern. The government policy factor $g_{t}$ exhibits a hump shape that peaks around the two year maturity. The covariance term $\mathbb{C}\left(A_{t}, g_{t}\right)$ is also contributing significantly to the hump. Its magnitude is determined by two factors, by the correlation between government policy uncertainty and productivity $\left(\rho^{A g}<0\right)$ and by the impact of government policy uncertainty on productivity $(\lambda<0)$. The impact of the production variance $\mathbb{V}\left[A_{t}\right]$ as well as of monetary policy uncertainty is monotonically decreasing in $\tau$. The latter factor has only little impact. From Corollary 1 and Figure 4 we can deduce the following:

Proposition 7 (Policy Uncertainty and the term structure of bond yield volatility).

- Government $g_{t}$ and monetary $m_{t}$ policy uncertainty raise the level of bond yield volatility. However, the effect is much stronger for fiscal as opposed to monetary policy uncertainty, i.e.

$$
\frac{b_{g}^{2}(\tau)}{\tau^{2}} \mathbb{V}\left[g_{t}\right]>\frac{b_{g}^{2}(\tau)}{\tau^{2}} \mathbb{V}\left[m_{t}\right]
$$

- The function $F^{g}(\tau)=\frac{b_{g}^{2}(\tau)}{\tau^{2}} \mathbb{V}\left[g_{t}\right]$ is hump-shaped across maturities. This implies that the effect of government policy uncertainty is highest for about 2 years maturity according to Figure 4.

The first result in Proposition 7 is straight-forward. Since clearly $\frac{b_{g}^{2}(\tau)}{\tau^{2}} \mathbb{V}\left[g_{t}\right]>0,\left(\frac{b_{m}^{2}(\tau)}{\tau^{2}} \mathbb{V}\left[m_{t}\right]>0\right)$ and $\mathbb{C}\left(A_{t}, g_{t}\right)>0$ adding the government (monetary) policy uncertainty factor raises the level of volatility. The second statement above is far less obvious to see, as it crucially depends on the parameter specifications of the model. Therefore, in Panels A through F of Figure 5, we explore in more detail the parameters responsible for generating the hump shape.

[Figure 5 about here]

As Panel A illustrates, the term structure of bond yield volatility exhibits a fundamentally different shape whenever $\lambda$ is negative or when $\lambda$ is set to zero, in which case government policy uncertainty does not affect the drift of productivity. Not only is the level of the term structure significantly higher whenever $\lambda<0$ than compared to the case when $\lambda=0$ but also, bond yield volatility becomes hump-shaped in time to maturity. Panels B and C show that the speed of mean 
reversion level $\kappa_{g}$ and volatility $\sigma_{g}$ have a similar effect on bond volatility. A more persistent and a highly volatile uncertainty process generate not only to an upward shift of the bond volatility term structure, but also a hump shape. ${ }^{22}$ Similar to Panel B, we observe in Panel D that a more permanent shock in productivity (decrease in $\kappa_{A}$ ) also accentuates the hump shape. In contrast, increasing production volatility $\sigma_{A}$ shifts the volatility term structure upward such that it eventually becomes monotonically decreasing (Panel E). This follows because the factor $\frac{b_{A}^{2}(\tau)}{\tau^{2}} \mathbb{V}\left(A_{t}\right)$ increases proportionally in $\sigma_{A}^{2}$ and since $\frac{b_{A}^{2}(\tau)}{\tau^{2}}$ is monotonically decreasing. Finally, Panel $\mathrm{F}$ shows that bond volatility is highly sensitive to changes in either $\eta_{1}$ or $\eta_{2}$. When the central bank becomes less responsive to deviations of the long term real target $\bar{k}$, the level of bond volatility decreases substantially. In contrast, if the central bank becomes less responsive to deviations of the long term nominal target $\bar{\pi}$, bond volatility increases significantly. ${ }^{23}$

\section{Empirical analysis}

Our theoretical model gives rise to several theoretical predictions. In the subsequent empirical analysis, we examine the following four testable hypotheses.

Hypothesis 1 (H1): Our first prediction can be deduced directly from Proposition 5, which implies that nominal yields fall when either government or monetary policy uncertainty increases and vice versa. From Proposition 5, this effect is mainly driven by government policy uncertainty (see Equation (31)).

Hypothesis 2 (H2): Higher (lower) fiscal or monetary policy uncertainty increases (decreases) nominal yield volatility (see Equation (39) in Proposition 7). This effect is again mainly driven by government policy uncertainty.

\footnotetext{
${ }^{22}$ The long run mean of government policy uncertainty $\theta_{g}$ increases yield dispersion proportionally, since $\frac{\partial \mathbb{V}(Y(t, \tau)}{\partial \theta_{g}}>0$. Hence, the long term mean does not contribute to a hump shape.

${ }^{23}$ Without providing the corresponding plots, we remark that whenever both $\eta_{1}$ and $\eta_{2}$ are both reduced, there will be a parallel downward shift in the level of bond yield volatility. Furthermore, the impact of risk aversion on the term structure of bond volatilities is less pronounced and leads to an almost parallel downward shift of the term structure.
} 
Hypothesis 3 (H3): From the second result in Proposition 7, the hump shape of the term structure of bond yield volatility is mainly driven by government policy uncertainty and not by monetary policy uncertainty.

Hypothesis 4 (H4): From Equation (37), bond risk premia are affected by both monetary and government policy uncertainty.

To investigate the joint effect of government and monetary policy uncertainty on the yield curve, its term structure of volatility, and on risk premia, we use as a proxy for both types of policy uncertainties, the economic policy uncertainty (EPU) index developed by Baker et al. (2012). Thus, in our model this index would be approximately equivalent to setting $E P U_{t} \approx g_{t}+m_{t}$. In what follows, we will empirically test the above hypotheses by regressing nominal yields and yield volatility on the EPU index and our time series of government and monetary policy as well as a set of control variables.

\subsection{Data}

We obtain monthly Treasury Bill yields with maturity one, two, three, five, seven, and ten years from the Federal Reserve Board ranging from January 1990 until July 2014, from which we bootstrap the zero-coupon yield curve treating the treasury yields as par yields. From Datastream, we collect monthly data on a total of 2 macro variables, which we aggregate into a real business cycle activity factor and an inflation factor. ${ }^{24}$ As a measure for real activity we use industrial production (IP). As an inflation factor, we use the consumer price index (CPI). We then compute monthly log-growth rates over one year for each of the macro control variables.

As an alternative to the EPU, we proxy for (policy) uncertainty using the VIX index. As a measure for economic condition we include the Chicago Fed National Activity Index (CFNAI), which we obtain from the FRED database (St. Louis Fed). ${ }^{25}$ We also use treasury bond implied volatility

\footnotetext{
${ }^{24}$ Similar control variables have also been used by Ang \& Piazzesi (2003), Evans \& Marshall (2007), Ludvigson \& $\mathrm{Ng}$ (2009) or Joslin, Priebisch \& Singleton (2014) in their study of the economic determinants of the term structure of nominal interest rates.

${ }^{25}$ The reason why we include the CFNAI regressor is to test whether either monetary or government policy uncertainty has predictive power after controlling for the state the economy is in. This is because it is reasonable to assume that uncertainty about the government's future policy choice is in general larger in weaker economic conditions.
} 
(TIV) based on weighted average of one-month options on treasury bonds with maturity of two, five, ten, and 30 years as a proxy for bond market volatility.

In addition we collect two time series, which we refer to as Financial Variables' (FV). They include the monthly log growth rate of the S\&P composite dividend yield index (DY), which has been shown to have forecasting power by Fama \& French (1989), and the term spread measured as the ten-year yield less the federal funds rate (TS).

\subsection{Construction of government and monetary policy uncertainty index}

The economic policy uncertainty (EPU) index developed by Baker et al. (2012) has been recently used by a number of studies. ${ }^{26}$ The EPU index is constructed from three main components, namely a news impact part which is based on news paper discussing economic policy uncertainty, a component that summarizes reports by the Congressional Budget Office (CBO) that compile lists of temporary federal tax code provisions, and a third component called 'economic forecaster disagreement', which draws on the Federal Reserve Bank of Philadelphia's Survey of Professional Forecasters and summarizes data on consumer price forecast dispersion and predictions for purchases of goods and services by state, local and federal government. ${ }^{27}$

For our setting, we need to decompose the EPU index into uncertainty related to government and monetary policy. To disentangle the two types of uncertainties, we use the 'categorical EPU data', which contains time-series on uncertainty related to government and monetary policy as well as further categorical variables. ${ }^{28}$ For our measure of government policy uncertainty, we extract the time-series 'fiscal policy', 'taxes', and 'government spending'. Furthermore, we argue that disagreement about temporary federal tax code provisions and forecast variation in local state and federal purchases of goods and services are sources of government policy uncertainty, whereas disagreement

\footnotetext{
${ }^{26}$ For instance, Pastor \& Veronesi (2013) show that government policy uncertainty carries a risk premium, and that stocks are more volatile and more correlated in times of high uncertainty. Brogaard \& Detzel (2012) use the same index and find that economic policy uncertainty forecast future market excess returns. Similarly, Gulen \& Ion (2012) show that policy-related uncertainty is negatively correlated with firm and industry level investment. When policy uncertainty increases firm's tend to reduce their investment.

${ }^{27}$ As Kelly et al. (2013) argue, it is difficult isolate exogenous variation in political uncertainty as it likely depends on various factors such as overall macro uncertainty. Therefore, the EPU index may not only capture government related uncertainty, but can be interpreted as a broader measure of uncertainty about economic fundamentals.

${ }^{28}$ This data is also available from http://www.policyuncertainty.com/.
} 
about future inflation (CPI disagreement) can be related to monetary uncertainty.

Therefore, in order to construct our index of 'government policy uncertainty' labeled as 'GPU' we include the time series 'fiscal policy', 'Taxes', 'Government', 'FedStateLocal Ex disagreement' and 'Tax expiration'. We place half of the weight to the time series 'fiscal policy' and the other half is equally distributed among the time series 'Taxes', 'Government', 'FedStateLocal Ex disagreement' and 'Tax expiration'.29 Our measure of 'monetary policy' uncertainty, which we abbreviate by 'MPU', consists of the time series 'monetary policy uncertainty' and 'CPI disagreement' of which each obtain weight $1 / 2$.

\subsection{Policy uncertainty and the yield curve}

To investigate the relationship between the yield curve and the EPU index, we make the following regression,

$$
Y(t, \tau)=\beta_{0}+\beta_{1} P U_{t}+\epsilon_{t}, \quad P U_{t} \in\left\{E P U_{t}, G P U_{t}, M P U_{t}\right\}
$$

where $P U_{t}$ denotes either the EPU, GPU, or MPU index at time $t$, and $\epsilon_{t}$ is the regression error term. ${ }^{30}$

According to hypothesis H1, we expect the coefficient in Equation (40) to be negative for all the three uncertainty indexes. Additionally, we run the same regression as in Equation (40) above, just replacing the $P U_{t}$ index with the VIX index. Since the VIX index is a generally accepted measure of overall economic uncertainty and positively correlated with the EPU index, ${ }^{31}$ we expect the regression coefficient to be negative as well. We also regress $Y(t, \tau)$ on both the VIX and the EPU index, and on the VIX together with the GPU and MPU indices. By doing so, we can identify those variables that exhibit greater predictive power.

In Table 3 we summarize the results for the EPU index. ${ }^{32}$ The first row labeled 'EPU' shows that

\footnotetext{
${ }^{29}$ We divided the time series 'Tax expiration' by a factor of ten, because its impact would otherwise pull the index up substantially at the end of the time series. Furthermore, as tax laws are only altered infrequently, the 'Tax expiration' remains constant over several months up to two years. Its unscaled inclusion would lead to an underestimation in policy uncertainty variation.

${ }^{30}$ To address potential concerns about robustness of our results, we compute following Newey \& West (1994) standard errors with five lags to account for heteroskedasticity and autocorrelation (HAC) in residuals.

${ }^{31}$ For our sample, the correlation coefficient between VIX and EPU is 0.45 .

${ }^{32}$ For all the regressions tables that follow the intercept estimate $\hat{\beta}_{0}$ and corresponding HAC errors are not displayed.
} 


\begin{tabular}{cccccccc}
\hline & $\tau$ & $1 \mathrm{Y}$ & $2 \mathrm{Y}$ & $3 \mathrm{Y}$ & $5 \mathrm{Y}$ & $7 \mathrm{Y}$ & $10 \mathrm{Y}$ \\
\hline \multirow{2}{*}{ EPU } & $\mathrm{EPU}$ & $-3.70^{* * *}$ & $-3.68^{* * *}$ & $-3.51^{* * *}$ & $-3.02^{* * *}$ & $-2.61^{* * *}$ & $-2.16^{* * *}$ \\
& $t_{E P U}$ & $(-7.08)$ & $(-6.95)$ & $(-6.61)$ & $(-5.67)$ & $(-4.95)$ & $(-4.19)$ \\
& $R_{a d j}^{2}$ & 0.29 & 0.29 & 0.28 & 0.24 & 0.21 & 0.17 \\
\hline \multirow{2}{*}{ VIX } & VIX & -0.04 & $-0.05^{*}$ & $-0.05^{*}$ & $-0.05^{*}$ & $-0.04^{*}$ & $-0.04^{*}$ \\
& $t_{V I X}$ & $(-1.50)$ & $(-1.67)$ & $(-1.76)$ & $(-1.86)$ & $(-1.78)$ & $(-1.91)$ \\
& $R_{a d j}^{2}$ & 0.02 & 0.02 & 0.02 & 0.02 & 0.02 & 0.03 \\
\hline \multirow{2}{*}{ EPU \& VIX } & EPU & $-4.07^{* * *}$ & $-4.00^{* * *}$ & $-3.78^{* * *}$ & $-3.19^{* * *}$ & $-2.75^{* * *}$ & $-2.13^{* * *}$ \\
& $t_{E P U}$ & $(-8.26)$ & $(-7.77)$ & $(-7.17)$ & $(-5.76)$ & $(-4.80)$ & $(-3.75)$ \\
& VIX & 0.04 & 0.03 & 0.03 & 0.02 & 0.01 & 0.01 \\
& $t_{V I X}$ & $(1.62)$ & $(1.37)$ & $(1.17)$ & $(0.75)$ & $(0.58)$ & $(0.15)$ \\
& $R_{a d j}^{2}$ & 0.30 & 0.30 & 0.29 & 0.24 & 0.21 & 0.16 \\
\hline
\end{tabular}

Table 3: The table displays slope coefficients of the regression of $Y(t, \tau)$ on $E P U_{t}, Y(t, \tau)$ on $V I X_{t}$, and $Y(t, \tau)$ on $E P U_{t}$ and $V I X_{t}$ for $\tau=1 \mathrm{Y}, 2 \mathrm{Y}, 3 \mathrm{Y}, 5 \mathrm{Y}, 7 \mathrm{Y}$, and $10 \mathrm{Y}$. Values in brackets below represent HACrobust $t$-statistics. $R_{a d j}^{2}$ refers to adjusted coefficient of determination. By ${ }^{* * *},{ }^{* *},{ }^{*}$ we denote $1 \%, 5 \%$, and $10 \%$ statistical significance, respectively.

higher policy uncertainty EPU reduces yields across all maturities, confirming our model hypothesis H1. The effect is highly significant at the $5 \%$ level and decreasing in $\tau$, indicating that the short end of the yield curve is more responsive to shocks in policy uncertainty than the long end. Furthermore, the adjusted $R^{2}$ indicate that the single factor EPU accounts for $29 \%$ of total variability at the short end of the yield curve. This value decreases monotonically to $18 \%$ at the long end of the curve.

The row 'VIX' in Table 3 shows that, similar to the EPU index, a rise in the VIX index also leads to a statistically significant decline in nominal yields along the entire yield curve. However, this relationship is statistically significant only at the $10 \%$ confidence level and insignificant at the short end of the yield curve. In addition, the impact of the VIX is substantially smaller for any $\tau$ as the impact of the EPU and GPU indexes. Moreover, its explanatory power is also considerably lower, with an $R^{2}$ between $2 \%$ and $3 \%$, compared to $R^{2}$ ranging between $18 \%$ to $29 \%$ for the EPU and $10 \%$ to $20 \%$ for the GPU.

The row 'EPU \& VIX' in Table 3 shows that the only significant predictor is the EPU index, as the regression coefficient for the VIX is statistically insignificant across all maturities, once both regressors are added to the regression equation. The same conclusion can be drawn from row 'GPU \& VIX' as government policy uncertainty remains the only statistically significant predictor along 
the entire term structure. Lastly, row 'MPU \& VIX' shows that the MPU index has no predictive power for any $\tau$.

To check for the robustness of our results, we add different controls to the regression equation. We add the economic condition (EC) controls 'CFNAI' and 'VIX'. Furthermore, we also include the financial variables (FV) factor and macro controls (MC) as discussed in Section 4.1.

\begin{tabular}{cccccccc}
\hline & $\tau$ & $1 \mathrm{Y}$ & $2 \mathrm{Y}$ & $3 \mathrm{Y}$ & $5 \mathrm{Y}$ & $7 \mathrm{Y}$ & $10 \mathrm{Y}$ \\
\hline \multirow{2}{*}{$\mathrm{EC}$} & $\mathrm{EPU}$ & $-4.14^{* * *}$ & $-4.05^{* * *}$ & $-3.83^{* * *}$ & $-3.24^{* * *}$ & $-2.78^{* * *}$ & $-2.17^{* * *}$ \\
& $t_{E P U}$ & $(-9.51)$ & $(-8.79)$ & $(-7.95)$ & $(-6.15)$ & $(-5.10)$ & $(-4.03)$ \\
& $R_{a d j}^{2}$ & 0.30 & 0.30 & 0.28 & 0.24 & 0.21 & 0.16 \\
\hline \multirow{2}{*}{$\mathrm{EC}+\mathrm{FV}$} & $\mathrm{EPU}$ & $-2.56^{* * *}$ & $-2.82^{* * *}$ & $-2.87^{* * *}$ & $-2.72^{* * *}$ & $-2.48^{* * *}$ & $-2.09^{* * *}$ \\
& $t_{E P U}$ & $(-4.07)$ & $(-4.27)$ & $(-4.23)$ & $(-3.91)$ & $(-3.62)$ & $(-3.22)$ \\
& $R_{a d j}^{2}$ & 0.47 & 0.40 & 0.35 & 0.26 & 0.22 & 0.17 \\
\hline \multirow{2}{*}{$\mathrm{CC}+\mathrm{FV}+\mathrm{MC}$} & $\mathrm{EPU}$ & $-2.75^{* *}$ & $-3.02^{* *}$ & $-3.07^{* *}$ & $-2.91^{* *}$ & $-2.66^{* *}$ & $-2.28^{* *}$ \\
& $t_{E P U}$ & $(-5.60)$ & $(-5.81)$ & $(-5.75)$ & $(-5.47)$ & $(-5.14)$ & $(-4.73)$ \\
& $R_{a d j}^{2}$ & 0.62 & 0.56 & 0.52 & 0.47 & 0.44 & 0.42 \\
\hline \hline
\end{tabular}

Table 4: The table reports slope coefficients of the regression, $Y(t, \tau)$ on $E P U_{t}$ and EC controls (EC), $Y(t, \tau)$ on $E P U_{t}$ and $\mathrm{EC}, \mathrm{FV}$ variables $(\mathrm{EC}+\mathrm{FV}), Y(t, \tau)$ on $E P U_{t}$ and $Y(t, \tau)$ on $E P U_{t}$, and $\mathrm{EC}, \mathrm{FV}, \mathrm{MC}$ controls (Full Reg.) for $\tau=1 \mathrm{Y}, 2 \mathrm{Y}, 3 \mathrm{Y}, 5 \mathrm{Y}, 7 \mathrm{Y}$ and 10Y. Values in brackets below represent HAC-robust $t$-statistics. $R_{a d j}^{2}$ refers to adjusted coefficient of determination. By ${ }^{* * *},{ }^{* *},{ }^{*}$ we denote $1 \%, 5 \%$, and $10 \%$ statistical significance, respectively.

Table 4 shows that the EPU regression coefficient remains significant for any maturity and across all regressions. However, compared to Table 3, its impact is considerably reduced (primarily at the short end), especially when we include the financial variables (FV). The reason for this decline is because those regressors exhibit strong positive correlation with the EPU index. Not surprisingly, their estimated impact on contemporaneous yield changes is also negative. Their average is -4.62 (DY), and -0.16 (TS). Whereas the $R_{a d j}^{2}$ essentially stays the same after adding the CFNAI control (row 'EC'), it increases considerably, although mainly at the short-end of the yield curve, after adding the financial factors (see row 'EC+FV'). Lastly, as the row labeled 'EC,FV+MC' shows, that whereas adding macro controls to the regression equation does not impact the statistical significance and magnitude of the EPU index, it considerably increases the amount of explained variation as the $R_{a d j}^{2}$ increases substationally across every maturity. ${ }^{33}$

\footnotetext{
${ }^{33}$ This increase in $R_{a d j}^{2}$ is predominantly driven by inflation as it has a statistically significant, very large and positive
} 
[Figure 6 about here]

While Table 4 is concerned with the EPU index, we now turn our focus on the individual impact of government and monetary policy uncertainty. In Figure 6, we plot the estimated regression coefficients of the GPU and MPU index together with their 95\% HAC-robust confidence intervals. Panel A and B show that, whereas the impact of fiscal policy uncertainty remains negative and statistically significant after including all control variables (Panel B), the monetary policy uncertainty exhibits a positive and statistically significant effect on contemporaneous yield movements even after including all control variables (Panel B). This somewhat puzzling result indicates that this effect might be do to strong correlation with the GPU index or other control variables. Therefore, in order to separately analyze the impact of fiscal and monetary policy uncertainty, we regress yields on the GPU and MPU individually in Panel C and D (with controls). Whereas the negative and statistically significant effect of the GPU index on contemporaneous yields remains unchanged, the impact of MPU is insignificant for every maturity. These results suggest that, the MPU's strong positive impact on yields is mainly do to its large positive correlation with the GPU index. Overall, we conclude that these results are in line with hypothesis $\mathbf{H 1}$ stated above, i.e. higher fiscal as opposed to monetary policy uncertainty decreases the nominal yields for every maturity.

\subsection{The term structure of bond yield volatility and policy risk}

Our theoretical results from Section 3.4 suggest that the inclusion of a time-varying government policy risk factor not only raises the level of the yield curve, but is also a key driver in generating the empirically observed hump shape of the bond volatility term structure. We now test these predictions using both the EPU, and our measures of government and monetary policy uncertainty including all the control variables from above.

Our measure for observed volatility is realized volatility as given in Equation (41) aggregated on a monthly level from business day data.

$$
\mathcal{V}_{t}(Y(t, \tau))=\sqrt{\sum_{d=1}^{D-1}\left(\log \left(\frac{Y(d+1, \tau)}{Y(d, \tau)}\right)\right)^{2}}, \quad Y(t, \tau), d \in\{1, \ldots, D-1\},
$$

impact on the term structure of nominal interest rates. 
where $D$ denotes the number of daily observations (about 20 business days per month) and $\tau=1 \mathrm{Y}, 2 \mathrm{Y}$, $3 \mathrm{Y}, 5 \mathrm{Y}, 7 \mathrm{Y}$ and $10 \mathrm{Y}$ to construct a time series of monthly realized bond yield volatility. In addition to our control variables from the previous sections, we also include treasury implied volatility (TIV) to proxy for fixed-income implied volatility. ${ }^{34}$

$$
\mathcal{V}_{t}[Y(t, \tau)]=\beta_{0}+\beta_{1} P U_{t}+\operatorname{cont}_{t}+\epsilon_{t}
$$

where $P U_{t}$ denotes either the EPU, GPU, or MPU index at time $t$, cont $t_{t}$ summarizes all the control variables and $\epsilon_{t}$ is the regression error term. From our hypothesis H2, we expect the sign of the regression coefficient in (42) of the EPU and the GPU index to be positive for all maturities. ${ }^{35}$ Furthermore, from hypothesis $\mathbf{H 3}$ we expect that economic or government policy uncertainty have a hump-shaped effect on the term structure of bond yield volatility. Thus, we should observe that the estimated regression coefficients peaks around the two year maturity as the realized bond volatility curve in Figure 3 does.

\begin{tabular}{cccccccc}
\hline & $\tau$ & 1 & 2 & 3 & 5 & 7 & 10 \\
\hline \multirow{2}{*}{ Simple } & EPU & $0.192^{* * *}$ & $0.194^{* * *}$ & $0.166^{* * *}$ & $0.121^{* * *}$ & $0.091^{* * *}$ & $0.063^{* * *}$ \\
& $t_{E P U}$ & $(7.18)$ & $(9.72)$ & $(8.88)$ & $(8.86)$ & $(7.66)$ & $(7.01)$ \\
& $R_{a d j}^{2}$ & 0.37 & 0.43 & 0.44 & 0.43 & 0.40 & 0.37 \\
\hline \multirow{2}{*}{ EC } & EPU & $0.192^{* * *}$ & $0.188^{* * *}$ & $0.156^{* * *}$ & $0.111^{* * *}$ & $0.079^{* * *}$ & $0.051^{* * *}$ \\
& $t_{E P U}$ & $(6.39)$ & $(9.37)$ & $(8.73)$ & $(8.60)$ & $(7.46)$ & $(6.67)$ \\
& $R_{a d j}^{2}$ & 0.39 & 0.44 & 0.45 & 0.45 & 0.43 & 0.43 \\
\hline \multirow{2}{*}{ EC +FV \& TIV } & EPU & $0.135^{* * *}$ & $0.135^{* * *}$ & $0.118^{* * *}$ & $0.087^{* * *}$ & $0.064^{* * *}$ & $0.042^{* * *}$ \\
& $t_{E P U}$ & $(4.08)$ & $(5.41)$ & $(4.99)$ & $(4.91)$ & $(4.18)$ & $(3.70)$ \\
& $R_{a d j}^{2}$ & 0.44 & 0.50 & 0.50 & 0.48 & 0.45 & 0.44 \\
\hline \multirow{2}{*}{ Full Regression } & $\mathrm{EPU}$ & $0.146^{* * *}$ & $0.145^{* * *}$ & $0.125^{* * *}$ & $0.095^{* * *}$ & $0.071^{* * *}$ & $0.04^{* * *}$ \\
& $t_{E P U}$ & $(4.27)$ & $(6.24)$ & $(5.76)$ & $(6.62)$ & $(5.90)$ & $(5.29)$ \\
& $R_{a d j}^{2}$ & 0.47 & 0.56 & 0.57 & 0.57 & 0.57 & 0.56 \\
\hline
\end{tabular}

Table 5: The table displays slope coefficients of the regression of $\mathcal{V}_{t}[Y(t, \tau)]$ on $E P U_{t}(\mathrm{EPU}), \mathcal{V}_{t}[Y(t, \tau)]$ on $E P U_{t}$ and EC controls (EC), $\mathcal{V}_{t}[Y(t, \tau)]$ on $E P U_{t} \mathrm{EC}$ and $\mathrm{FV}$ variables $(\mathrm{EC}+\mathrm{FV})$ including the TIV and $\mathcal{V}_{t}[Y(t, \tau)]$ on $E P U_{t}, \mathrm{EC}, \mathrm{FV}$ (including TIV) and MC controls (Full Regression). The yield maturities are 1, $2,3,5,7$, and 10 years. Values in brackets below represent HAC-robust $t$-statistics. $R_{a d j}^{2}$ refers to adjusted coefficient of determination. By ${ }^{* * *},{ }^{* *},{ }^{*}$ we denote $1 \%, 5 \%$, and $10 \%$ statistical significance, respectively.

\footnotetext{
${ }^{34}$ Treasury bond implied volatility is based on weighted average of very liquid one-month options on treasury bonds with maturity 2, 5, 10, and 30 years. To obtain monthly data, we compute the sample mean using daily observations.

${ }^{35}$ From our theoretical analysis from Section 3 and 3.4, the contemporaneous impact of monetary policy uncertainty is marginal and therefore we do not expect to find any significant estimates for the MPU index.
} 
We report the results of the regression in Equation (42) in Table 5. We find our hypothesis $\mathbf{H 2}$ concerning the EPU confirmed. Higher economic policy uncertainty increases bond volatility for any maturity. Once we include the financial control variables, the impact of the EPU index is reduced for any maturity and the explanatory power increases from an average $\bar{R}_{a d j}^{2}=0.40$ to $\bar{R}_{a d j}^{2}=0.47$. This observation suggests, similar to the results in Table 4, that financial factors are also important predictors of bond variance. Analyzing in more detail, we see from row 'Full Regression' that there is only a moderate increase in predictive power once we also include our macro controls.

Concerning hypothesis H3, although the impact of economic policy is highly statistically significant, it gradually declines along the entire term structure. Hence, the EPU index is not able to generate a hump in the volatility term structure. Therefore, if we would use the EPU as our proxy for government uncertainty, this result in conflict with our hypothesis H3. However, since in the EPU index government and monetary uncertainty factors are intermingled, we need to analyze the impact of the government and monetary policy uncertainty index separately.

[Figure 7 about here]

Figure 7 shows that our hypotheses $\mathbf{H 2}$ and $\mathbf{H 3}$ are supported by the data. Not only does realized volatility rise when government policy uncertainty increases, its effect is also hump-shaped with a peak around a maturity of two years. Similar to the yield regressions from above, once we add all control variables, the estimated impact of the GPU is substantially reduced, yet it remains statistically significant and hump-shaped (Panel B). Moreover, similar to above, we find that the statistical significance of the MPU is misleading. In Panel $\mathrm{C}$ we regress realized volatility on the GPU and MPU index individually (Panel D is with controls). Including only the GPU or the MPU shows that, whereas the GPU remains positive, significant and hump-shaped in time to maturity, the MPU index changes signs (compared to Panel A or B) and is no longer significant at the 5\% confidence level for all maturities. 


\subsection{Bond risk premia}

Hypothesis $\mathbf{H} 4$ states that both government and monetary policy uncertainty should explain bond risk premia. To explore the predictive power of the EPU index as well as our government and monetary policy indexes on future bond excess returns, we denote by

$$
r_{t+1}^{E, \tau_{i}}:=\log \left(B\left(t+1, \tau_{i}-1\right)\right)-\log \left(B\left(t, \tau_{i}\right)\right)-Y(t, 1)
$$

the log-excess return from buying at time $t$ a bond with time-to-maturity $\tau_{i}$ and selling it after one period. Furthermore, the log forward rate a time $t$ for entering contracts between time $t+\tau_{i}-1$ and $t+\tau_{i}$ is given by $F\left(t, \tau_{i}\right)=\log \left(B^{N}\left(t, \tau_{i}-1\right) / B^{N}\left(t, \tau_{i}\right)\right)$. The literature on bond risk premia usually compares the predictive power of a new predictor variable against the routinely used Cochrane \& Piazzesi (2005) factor (CP), which is constructed based on a tent-shaped linear combination of forward rates. ${ }^{36}$ Furthermore, from the covariance matrix of yields we extract the first three principal components which are commonly referred to as 'level', 'slope' and 'curvature' (see Litterman \& Scheinkman (1991)) As a further set of control variables, we also include the economic condition, the financial variables (without) and the set of macroeconomic factors as above.

[Figure 8 about here]

To analyze the forecasting power of the GPU und MPU index for the bond risk premium, we plot in Figure 8 the corresponding regression coefficients. In Panel A, we jointly regress bond excess bond returns GPU and the MPU in a single regression equation. In line with the factor loadings displayed in Panel A of Figure 4, both the GPU and the MPU load positively on the risk premium except for the MPU, which loads slightly negatively at the ten-year maturity. However, the loading of the MPU is statistically insignificant at all maturities. In contrast, the regression coefficient of the GPU is statistically significant at medium to longer maturities. Panel B shows that once we control for PCA and CP factors, economic condition, financial variables, and macro factors, the significance of the GPU is still preserved at every maturity, but its magnitude is significantly reduced. On the other hand, the MPU becomes also significant for all maturities and its slope exceeds the one of

\footnotetext{
${ }^{36}$ A detailed description of the construction of this factor is given in Cochrane \& Piazzesi (2005). To avoid collinearity problems, we only include the current one year yield $Y(t, 1)$ and the five- and ten year forward rates and we do not restrict the regression coefficients to sum up to one.
} 
the GPU factor. The adjusted $R^{2}$ after having included all regression controls averages some $89 \%$ across all maturities. When we only use the GPU and the MPU, however, the $R^{2}$ is just some $3 \%$ for the two-year maturity, but rises to $30 \%$ at the ten-year maturity. Analyzing the individual impact of the GPU and MPU respectively, we conclude that both are statistically significant and positive predictors of bond risk premia. However, including all our control variables shows that bond excess returns only load statistically significant on the MPU index and not on the GPU index, although its impact remains increasing in time to maturity just as in Panel B above. ${ }^{37}$ Hence, overall our hypothesis $\mathbf{H} \mathbf{4}$ is confirmed in that both government and monetary policy uncertainty have a positive significant impact on bond risk premia when added together to the regression equation.

\section{Conclusion}

We present a tractable dynamic equilibrium model of the term structure of interest rates with government (real) and monetary policy uncertainty shocks. Consistent with the data, our model is able to reproduce the flight-to-quality behavior, i.e., the observation that investors tend to increase their bond holdings in times of higher economic or government policy uncertainty and thereby lowering treasury bond yields. We calibrate our model to data and provide a detailed analysis of the dependence of the nominal yield curve and its corresponding term structure of nominal bond yield volatility on the structural model parameters. Even though our model belongs to the class of affine term structure models, it is capable of reproducing the empirically observed hump-shape of the term structure of bond volatility. To achieve this result, two key feature in our model are essential. First, that the long run growth path of productivity is time-varying and negatively dependent on government policy uncertainty. Secondly, that government policy uncertainty affects the nominal side of the economy whenever the central bank responds actively to deviations of output and inflation growth from their respective target rates.

Even though our simple empirical analysis has only illustrative character, it sheds some light on the relationship between contemporaneous movements of the yield and bond yield curve in response

\footnotetext{
${ }^{37}$ If we exclude the CP factor from the regression, the GPU index becomes a statistically significant predictor of bond risk premia.
} 
to shocks in government and monetary policy uncertainty. Our empirical results support our modelimplied predictions that, first, increased policy uncertainty leads to an increased demand in bonds and therefore reducing their yields and, second, that higher economic policy uncertainty not only raises bond volatility but is also a key driver of generating the empirically observed hump-shaped structure of the bond yield volatility curve. Furthermore, by decomposing the EPU index into its components, we identify government policy uncertainty as the main culprit for the negative relationship between policy uncertainty and nominal yields and for the empirically observed high and often hump-shaped term structure of bond yield volatility. The explanatory power of the monetary policy uncertainty index for contemporaneous yields and bond volatility movements is mixed at best and mostly insignificant. However, monetary policy uncertainty is not irrelevant for the term structure of yields. We find it to be a key predictor for bond risk premia at any maturity, while government policy shocks lose some of their statistical significance once all controls are added to the regression equation. 
Panel A: Term Structure and Policy Uncertainty

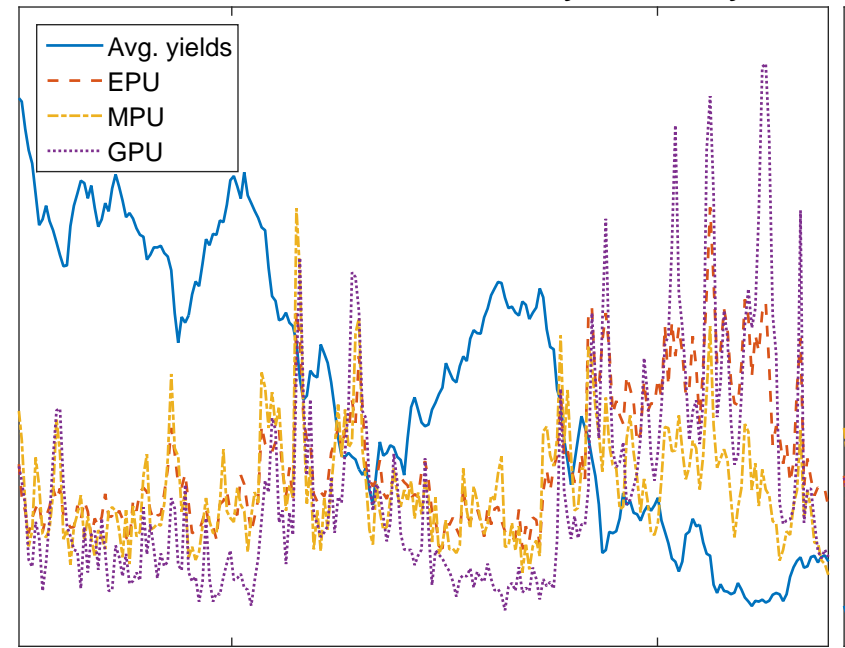

Jan00

$\operatorname{Jan} 10$
Panel B: Volatility and Policy Uncertainty

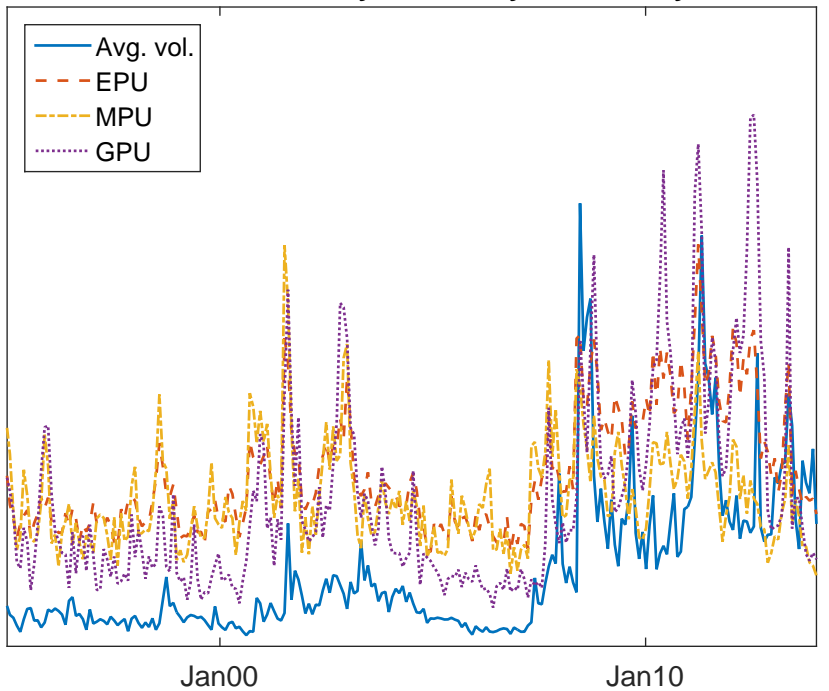

Figure 1: US Treasury bond yields (Panel A) and realized yield volatility (Panel B) with maturity $\tau=1 \mathrm{Y}$, $2 \mathrm{Y}, 3 \mathrm{Y}, 5 \mathrm{Y}, 7 \mathrm{Y}$ and $10 \mathrm{Y}$ and the economic policy uncertainty (EPU) index as constructed by Baker et al. (2012) plus the government (GPU) and monetary policy (MPU) index. Sample period ranges from January 1995 until June 2014. All indexes are scaled to match the scale of the Treasury bond yields and volatilities.
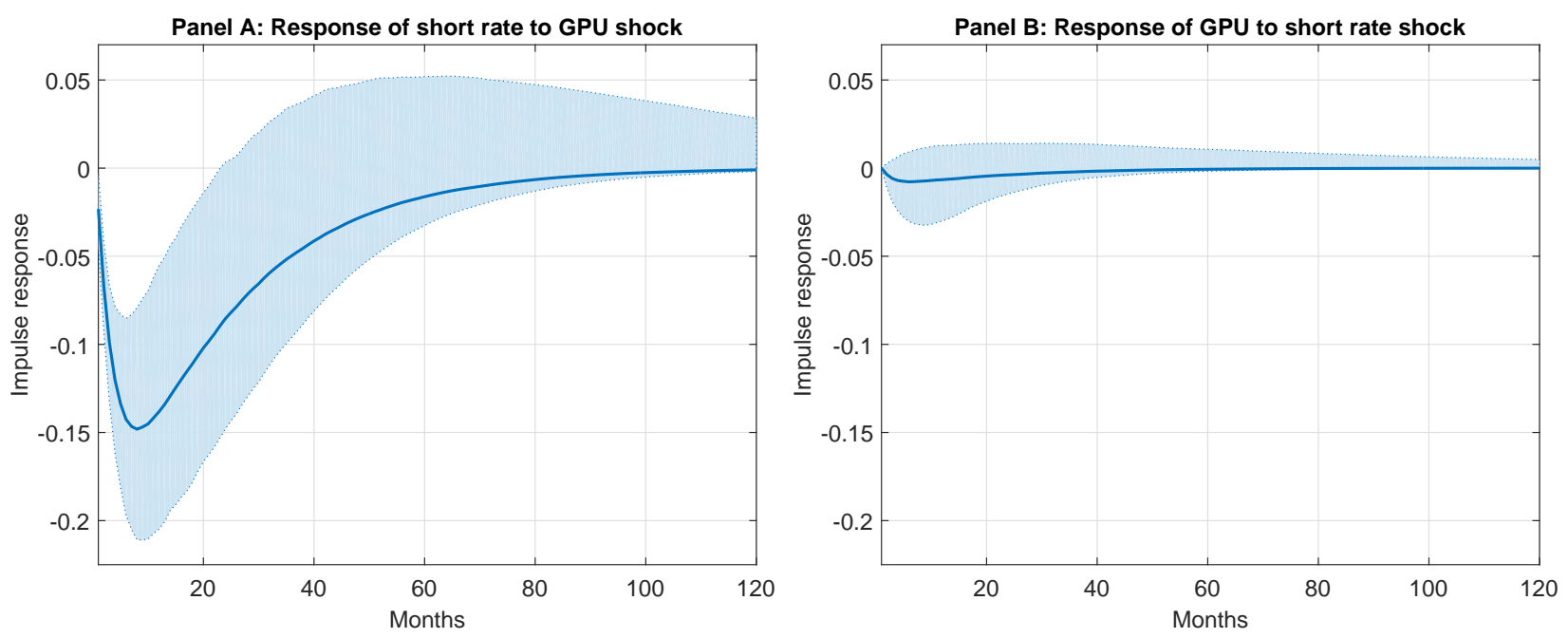

Figure 2: The figure plots the impulse response functions of a shock to the GPU on the short rate (Panel A) and a shock to the short rate on the GPU (Panel B). The short rate is approximated by the three-month T-bill rate. The impulse response functions are based on a VAR model including the GPU, MPU, and the three-month T-bill rate. The data sample spans the period from January 1990 to June 2014. The shaded are corresponds to the $95 \%$ confidence interval., 

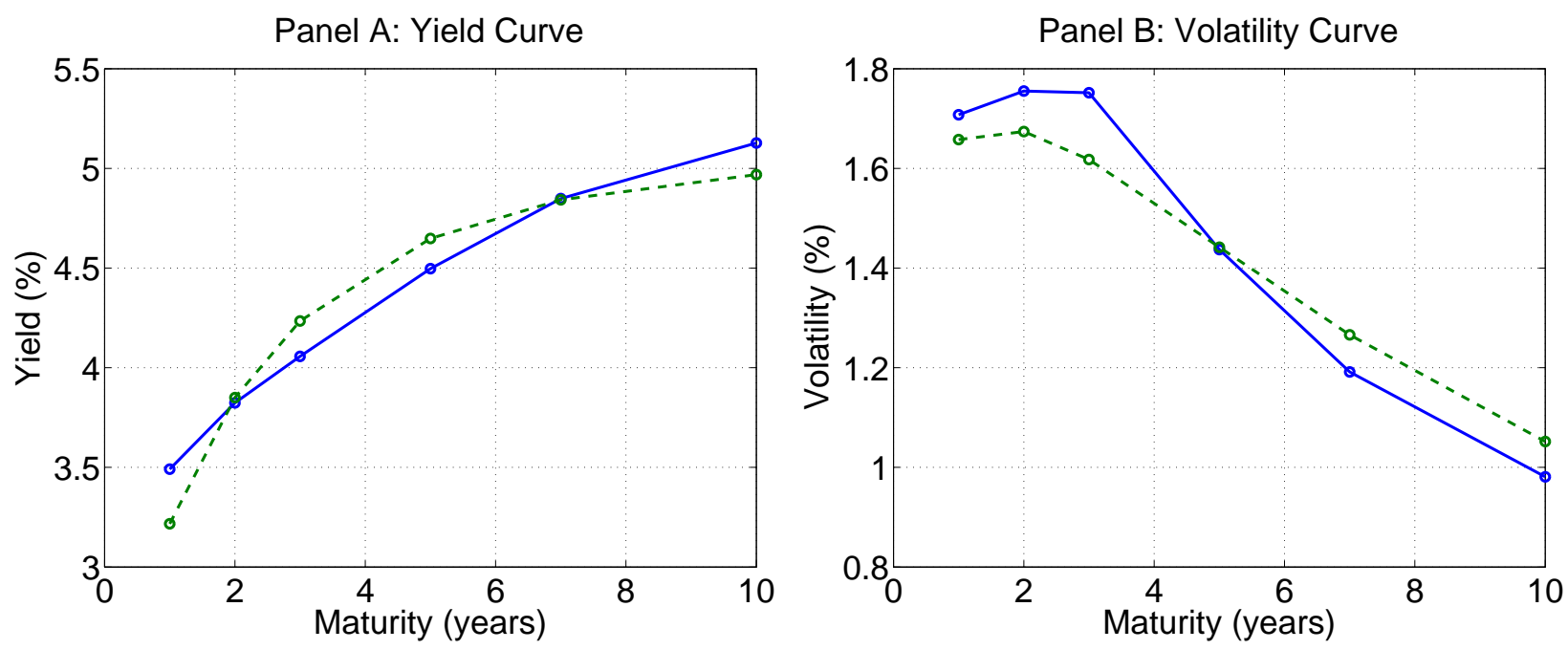

Figure 3: Empirical and fitted affine yield curve model. In Panel A we plot the empirical unconditional nominal yield curve based on monthly zero-coupon bonds (solid line) with the model implied yield curve (dashed line). Panel B compares the model-implied bond volatility term-structure (dashed line) to the empirical unconditional realized volatility term structure (solid line). Unconditional realized volatility is computed using monthly log-yield changes as described in Equation (41) with maturities of one, two, three, five, seven and ten years. Parameter values are given in Table 1 and 2 , respectively.
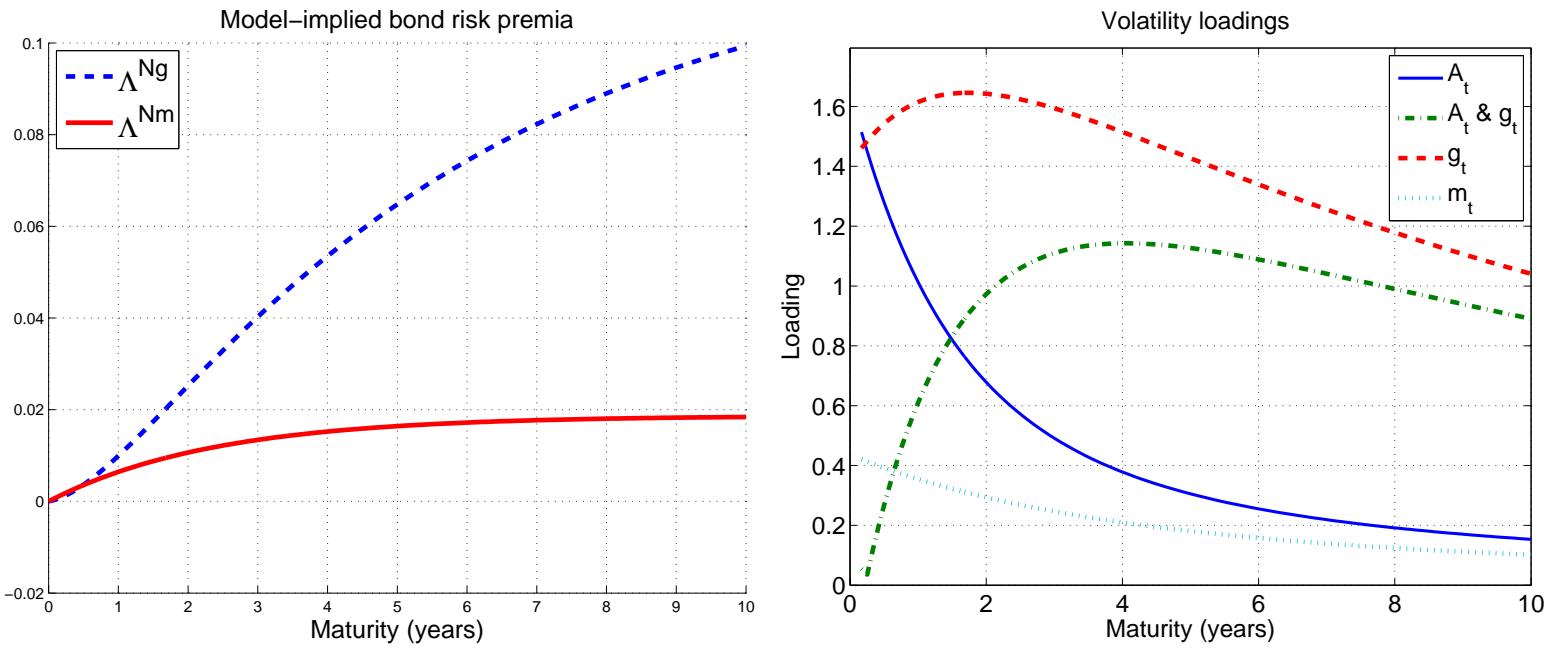

Figure 4: Loadings for bond risk premia and bond volatility. Panel A shows the different loadings of the government (dashed line) and monetary (solid line) policy uncertainty factors in the bond risk premium in Equation (37). Panel B shows the different loadings to total yield variance $\mathbb{V}[Y(t, \tau)]$ in Equation (38) of the productivity factor variance $\mathbb{V}\left[A_{t}\right]$ (solid line), government policy uncertainty factor variance $\mathbb{V}\left[g_{t}\right]$ (dashed line), the cross term $\mathbb{C}\left[A_{t}, g_{t}\right]$ (dash-dotted line) and the monetary policy uncertainty factor variance $\mathbb{V}\left[m_{t}\right]$ (dotted line). 

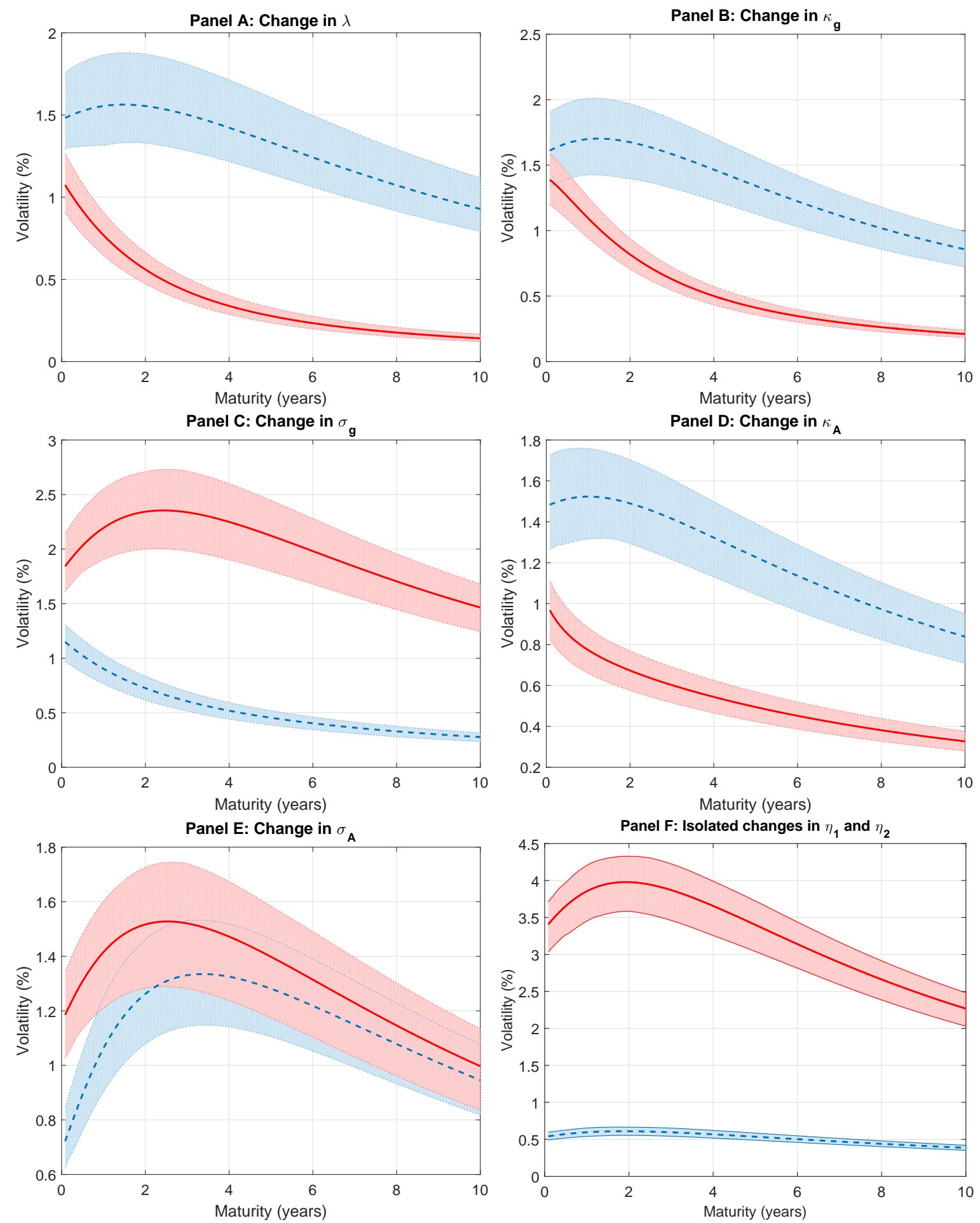

Figure 5: Volatility curve sensitivities. In Panel A, we plot the volatility term structure for $\lambda=0$ (solid line) and $\lambda=-1$ (dashed line). In Panel B, we increase $\kappa_{g}$ from 0.3 (dashed line) to 3 (solid line) and in Panel C we increase $\sigma_{g}$ from 0.05 (dashed line) to 0.5 (solid line). In Panel D, we plot the volatility curve for $\kappa_{A}=0.75$ (dashed line) and $\kappa_{A}=2.5$ (solid line) and Panel E shows the volatility curve for $\sigma_{A}=0.15$ (dashed line) and $\sigma_{A}=0.5$ (solid line). In Panel $\mathrm{F}$, we analyze the impact of changing $\eta_{1}$ and $\eta_{2}$. The dashed (solid) line represents the case when we leave $\eta_{2}\left(\eta_{1}\right)$ unchanged while reducing $\eta_{1}\left(\eta_{2}\right)$ by $20 \%$. All other parameters are as in Table 2. Shaded areas correspond to 95\%-confidence bound of the estimated yield curves. 

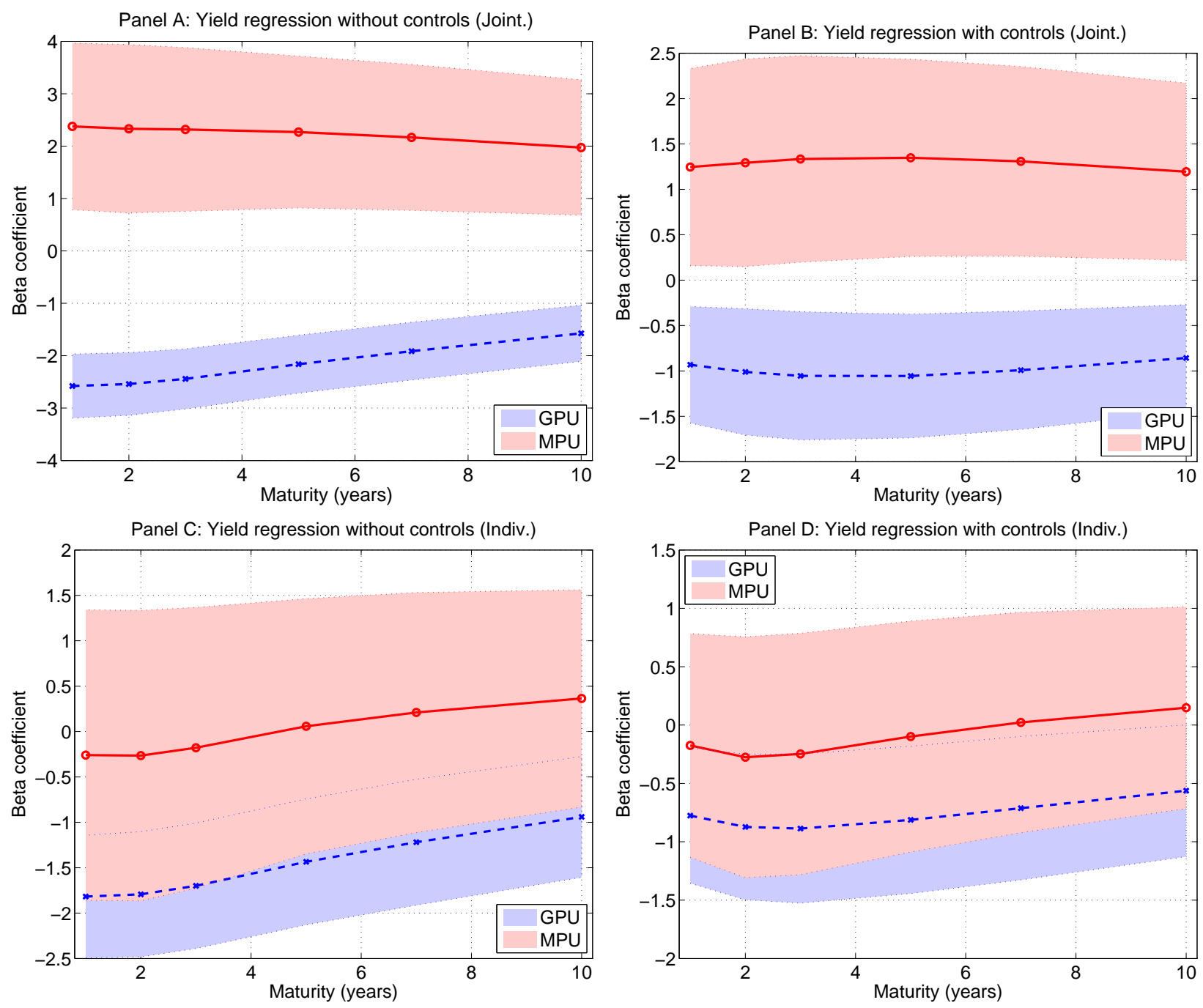

Figure 6: Yield curve regressions. Panel A displays the slope coefficients of the joint regression of yields $Y(t, \tau)$ on both the GPU (dashed line) and MPU (full line). In Panel B, we regress jointly $Y(t, \tau)$ on the GPU (dashed line) and on the MPU (full line) including the controls EC, FV and MC. Similarly in Panel C we display the slope coefficients of the individual regression of $Y(t, \tau)$ on only the GPU (dashed line) and $Y(t, \tau)$ on only the MPU (full line). In Panel D, we regress individually $Y(t, \tau)$ on the GPU (dashed line) and $Y(t, \tau)$ on the MPU (full line) including the controls EC, FV and MC for each regression. The yield maturities are 1, $2,3,5,7$, and 10 years. Shaded areas represent HAC-robust $95 \%$ confidence bounds. 

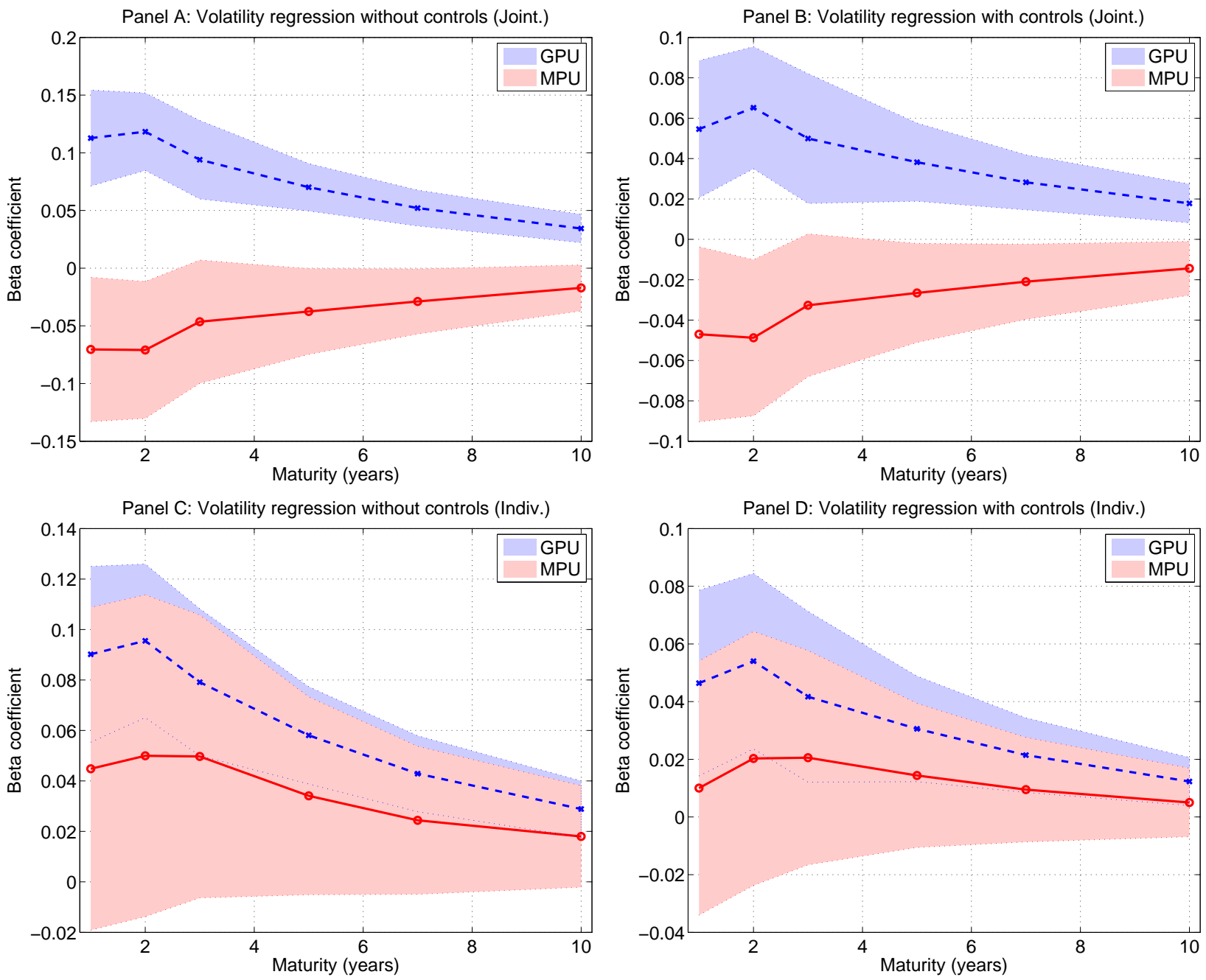

Figure 7: Yield volatility curve regressions. Panel A displays the slope coefficients of the joint regression of yield volatility $\mathcal{V}_{t}[Y(t, \tau)]$ on both the GPU (dashed line) and MPU (full line). In Panel B, we regress jointly $\mathcal{V}_{t}[Y(t, \tau)]$ on both the GPU (dashed line) and on the MPU (full line) including the controls EC, FV (with TIV) and MC. Similarly in Panel C we display the slope coefficients of the individual regression of $\mathcal{V}_{t}[Y(t, \tau)]$ on only the GPU (dashed line) and $\mathcal{V}_{t}[Y(t, \tau)]$ on only the MPU (full line). In Panel D, we regress individually $\mathcal{V}_{t}[Y(t, \tau)]$ on the GPU (dashed line) and $\mathcal{V}_{t}[Y(t, \tau)]$ on the MPU (full line) including the controls EC, FV (with TIV) and MC for each regression. The yield maturities are 1, 2, 3, 5, 7, and 10 years. Shaded areas represent HAC-robust $95 \%$ confidence bounds. 

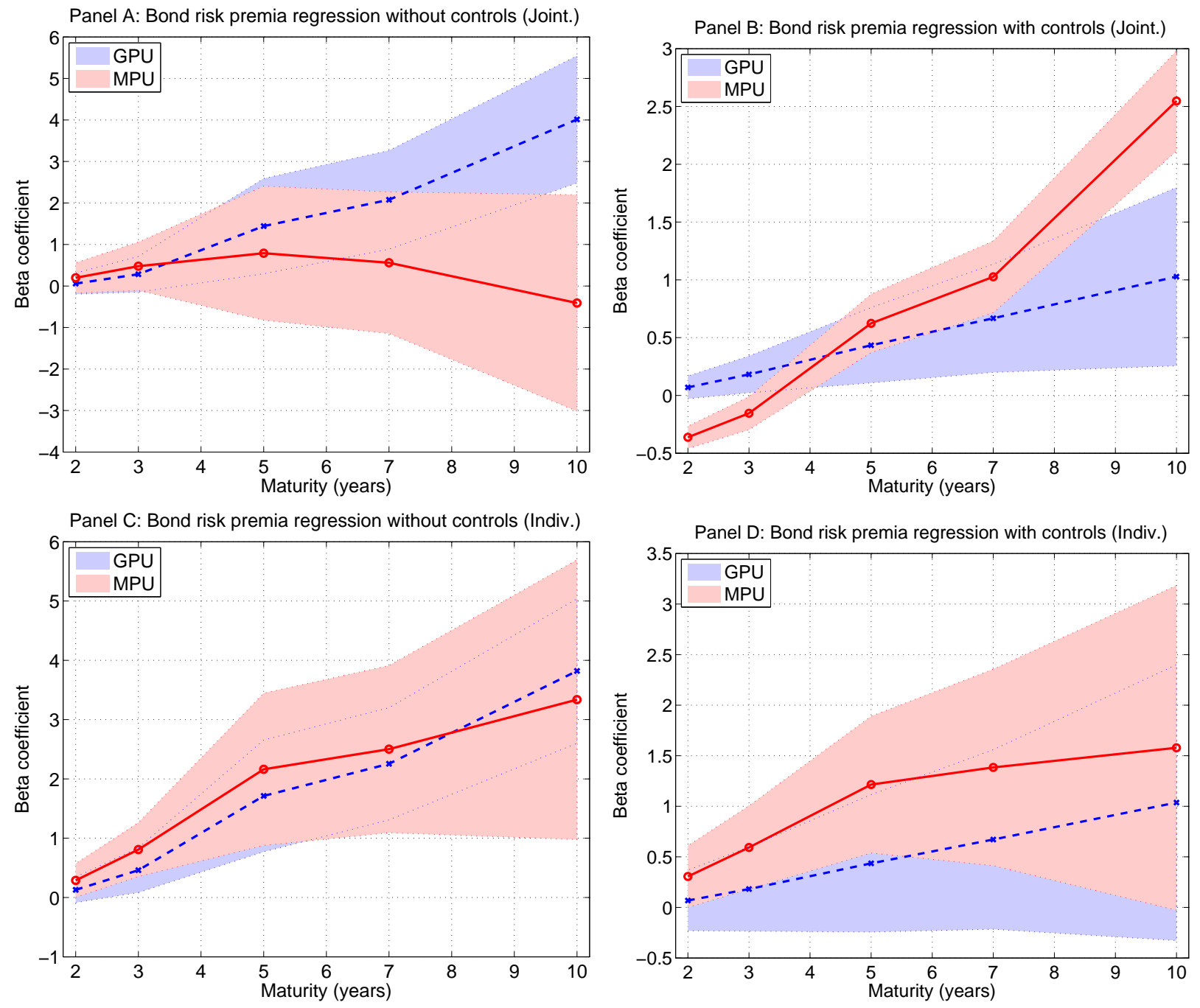

Figure 8: Bond risk premium regressions. Panel A displays the slope coefficients of the joint regression of the monthly excess returns $r_{t+1}^{E, \tau_{i}}$ on both the GPU (dashed line) and MPU (full line). In Panel B, we regress jointly $r_{t+1}^{E, \tau_{i}}$ on both the GPU (dashed line) and on the MPU (full line) including the controls CP, PCA, EC, FV and MC for each regression. Similarly in Panel C we display the slope coefficients of the individual regression of the monthly excess returns $r_{t+1}^{E, \tau_{i}}$ on the GPU (dashed line) and the monthly excess returns $r_{t+1}^{E, \tau_{i}}$ on the MPU (full line). In Panel D, we regress individually the monthly excess returns $r_{t+1}^{E, \tau_{i}}$ on the GPU (dashed line) and the monthly excess returns $r_{t+1}^{E, \tau_{i}}$ on the MPU (full line) including the controls CP, PCA, EC, $\mathrm{FV}$ and $\mathrm{MC}$ for each regression. The yield maturities are 1, 2, 3, 5, 7, and 10 years. Shaded areas represent HAC-robust $95 \%$ confidence bounds. 


\section{A Proofs}

\section{A.1 Proof of Proposition 1}

Proof. We start by deriving an $n$-th order recursive moment formula for the expectation of the government policy uncertainty process $g_{t}$ with dynamics

$$
d g_{t}=\kappa_{g}\left(\theta_{g}-g_{t}\right) d t+\sigma_{g} \sqrt{g_{t}} d W_{t}^{g}
$$

To compute its moments, let $f(g)=g^{n}$ where $n \in \mathbb{N}$, then an application of Itô's lemma and using Equation (A.1) gives

$$
\begin{aligned}
d g_{t}^{n} & =n g_{t}^{n-1} d g_{t}+\frac{1}{2} n(n-1) g_{t}^{n-2} \sigma_{g}^{2} g_{t} d t \\
& =\left(-n \kappa_{g} g_{t}^{n}+g_{t}^{n-1}\left(n \kappa_{g} \theta_{g}+\frac{n}{2}(n-1) \sigma_{g}^{2}\right)\right) d t+n g_{t}^{n-1} \sigma_{g} \sqrt{g_{t}} d W_{t}^{g}
\end{aligned}
$$

Integrating from $t$ to $T$, taking expectations on both sides, using Fubini's theorem and the law of iterated expectation, differentiating with respect to $T$ gives

$$
\begin{aligned}
& \psi_{t}^{\prime}(T)=\Upsilon_{0}(T)+\Upsilon_{1}(T) \psi_{t}(T) \\
& \Upsilon_{0}(T)=\mathbb{E}_{t}\left[g_{t+\tau}^{n-1}\right]\left(n \kappa_{g} \theta_{g}+\frac{n}{2}(n-1) \sigma_{g}^{2}\right) \\
& \Upsilon_{1}(T)=-n \kappa_{g}
\end{aligned}
$$

Conditional on $\kappa_{g}>0$, the solution to Equation (A.3) is

$$
\psi_{t}(t+\tau)=e^{\psi_{1} \tau} g_{t}^{n}+\int_{t}^{t+\tau} \Upsilon_{0}(t+\tau-u) e^{\Upsilon_{1}(t+\tau-u)} d u
$$

Then the first moment satisfies

$$
\begin{aligned}
\psi_{g}^{\prime}(t, T) & :=\frac{\mathbb{E}_{t}\left[g_{T}\right]}{d T}=\kappa_{g}\left(\theta_{g}-\mathbb{E}_{t}\left[g_{T}\right]\right) \\
\psi_{g}(t, t) & :=g_{t}>0
\end{aligned}
$$

where Equation (A.8) represents the initial condition. The solution this fist order linear differential equation in (A.7) can be represented as

$$
\psi_{g}(t, T):=\mathbb{E}_{t}\left[g_{T}\right]=\theta_{g}+\left(g_{t}-\theta_{g}\right) e^{-\kappa_{g} \tau}
$$


and similarly, using Equation (A.9) one obtains that the second moment is given by

$$
\begin{aligned}
\psi_{g^{2}}(t, T) & :=\mathbb{E}_{t}\left[g_{T}^{2}\right]=g_{t}^{2} e^{-2 \kappa_{g} \tau}+\frac{\left(e^{-\kappa_{g} \tau}-e^{-2 \kappa_{g} \tau}\right)\left(2 \theta_{g} \kappa_{g}+\sigma_{g}^{2}\right)}{2 \kappa_{g}} g_{t}+\frac{\theta_{g}\left(2 \theta_{g} \kappa_{g}+\sigma_{g}^{2}\right)\left(1-e^{-\kappa_{g} \tau}\right)^{2}}{2 \kappa_{g}} \\
\psi_{g^{2}}(t, t) & :=g_{t}^{2}>0
\end{aligned}
$$

from which one can immediately deduce that the variance of $g_{t}$ is

$$
\mathbb{V}_{t}\left[g_{T}\right]=\mathbb{E}\left[g_{T}^{2}\right]-\mathbb{E}\left[g_{T}\right]^{2}=\frac{\left(2 g_{t}\left(e^{-\kappa_{g} \tau}-e^{-2 \kappa_{g} \tau}\right)+\theta_{g}\left(1-e^{-\kappa_{g} \tau}\right)^{2}\right) \sigma_{g}^{2}}{2 \kappa_{g}}
$$

and its unconditional variance is

$$
\mathbb{V}\left[g_{t}\right]=\frac{\theta_{g} \sigma_{g}^{2}}{2 \kappa_{g}}
$$

Along the same line of argumentation, integrating Equation (5), applying Fubini's theorem and the law of iterated expectations we obtain that the conditional expected value of $A_{t}$ satisfies

$$
\begin{aligned}
& \psi_{A}^{\prime}(t, T):=\frac{\mathbb{E}_{t}\left[A_{T}\right]}{d T}=\kappa_{A}\left(\theta_{A}-\psi_{A}(t, T)\right)+\lambda \psi_{g}(t, T) \\
& \psi_{A}(t, t)=A_{t} \in \mathbb{R}
\end{aligned}
$$

where Equation (A.12) represents the initial condition for the process $A_{t}$. Using the expression for $\psi_{g}(t, T)$ in Equation (A.9), the solution can be obtained using standard methods. Passing to the limit, i.e., $T \rightarrow \infty$, gives the stationary expectation of $A_{t}$ in Proposition 1 . Next, in order to derive the second moment of the productivity process $A_{t}$, we have to first derive the an expression for the product expectation $\psi_{A g}(t, T):=\mathbb{E}_{t}\left[A_{t+\tau} g_{t+\tau}\right]$. An application of Itô's formula to $A_{t} g_{t}$ results in the following dynamics

$$
d\left(A_{t} g_{t}\right)=\left[\left(\kappa_{A} \theta_{A}+\rho^{A g} \sigma_{A} \sigma_{g}\right) g_{t}+\kappa_{A} \theta_{A} A_{t}-\left(\kappa_{A}+\kappa_{g}\right) A_{t} g_{t}+\lambda g_{t}^{2}\right] d t+\sigma_{A} g_{t}^{3 / 2} d W_{t}^{A}+\sigma_{g} g_{t}^{3 / 2} d W_{t}^{g}
$$

Then, as above, integrating from $t$ to $T=t+\tau$, applying Fubini's theorem and taking time $t$ conditional expectation shows that $\psi_{A g}(t, T)$ satisfies

$$
\begin{aligned}
\psi_{A g}^{\prime}(t, T) & :=\frac{\mathbb{E}_{t}\left[A_{T} g_{T}\right]}{d T}=\left(\kappa_{A} \theta_{A}+\rho^{A g} \sigma_{A} \sigma_{g}\right) \psi_{g}(t, T)+\kappa_{A} \theta_{A} \psi_{A}(t, T)-\left(\kappa_{A}+\kappa_{g}\right) \psi_{A g}(t, T)+\lambda \psi_{g^{2}}(t, T) \\
\psi_{A g}(t, t) & =A_{t} g_{t}
\end{aligned}
$$

where Equation (A.13) represents the initial condition. Solving this first order differential equation with time-dependent functions $\psi_{g}(t, T), \psi_{g^{2}}(t, T)$ and $\psi_{A}(t, T)$ gives the conditional covariance 
expression. Having obtained explicit conditional moments for $A_{t}, g_{t}$ and $A_{t} g_{t}$, the stationary covariance expression immediately follows from letting $T \rightarrow \infty$, i.e. $\lim _{T \rightarrow \infty}=\mathbb{C}_{t}\left(A_{t+\tau}, g_{t+\tau}\right)=$ $\mathbb{E}_{t}\left[A_{t+\tau}, g_{t+\tau}\right]-\mathbb{E}_{t}\left[A_{t+\tau}\right] \mathbb{E}_{t}\left[g_{t+\tau}\right]$. Finally, by similar arguments as above and an application of Itô's formula to $A_{t}^{2}$ we obtain that the second moment of $A_{t}$ satisfies

$$
\begin{aligned}
\psi_{A^{2}}^{\prime}(t, T) & :=\frac{\mathbb{E}_{t}\left[A_{T}^{2}\right]}{d T}=2 \kappa_{A}\left(\theta_{A}-\psi_{A}(t, T)\right)+2 \lambda \psi_{A g}(t, T)+\sigma_{A}^{2} \psi_{g}(t, T) \psi_{g^{2}}(t, T) \\
\psi_{A^{2}}(t, t) & =A_{t}^{2}
\end{aligned}
$$

Solving this ordinary first order differential equation with time-dependent coefficients gives the expression for the conditional expectation of $A_{t}$. From $\mathbb{V}_{t}\left(A_{t+\tau}\right)=\mathbb{E}\left[A_{t+\tau}^{2}\right]-\mathbb{E}\left[A_{t+\tau}\right]^{2}$ and letting $T \rightarrow \infty$ we immediately obtain the stationary variance expression for $A_{t}$.

\section{A.2 Proof of Proposition 3}

The optimal consumption and investment problem is

$$
\max _{C_{s}, M_{s}^{d}} \mathbb{E}_{t}\left[\int_{t}^{\infty} e^{-\beta s} U\left(C_{s}, M_{s}^{d}\right) d t\right]
$$

where $U\left(C_{t}, M_{t}^{d}\right)=\frac{1}{\gamma}\left(\left(C_{t}\left(M_{t}^{d}\right)^{\xi}\right)^{\gamma}-1\right)$ subject to the capital constraint in Equation (12). To simplify notation, let $X_{t}=\left(A_{t}, g_{t}\right)$ such that the value function is given by

$$
V=V\left(t, K_{t}, X_{t}\right)=\max _{\left\{C_{s}, M_{s}^{d}\right\}_{t \leq s<\infty}} \mathbb{E}_{t}\left[\int_{t}^{\infty} e^{-\beta s} U\left(C_{s}, M_{s}^{d}\right) d s\right]
$$

In equilibrium, there exists a value function $V\left(t, K_{t}, X_{t}\right)$ and control variables $\left\{C_{t}, M_{t}^{d}\right\}$ satisfying the HJB equation

$$
-\frac{\partial V\left(t, K_{t}, X_{t}\right)}{\partial t}=\max _{\left\{C_{t}, M_{t}^{d}\right\}}\left\{U\left(C_{t}, M_{t}^{d}\right)+\mathcal{A} V\left(t, K_{t}, X_{t}\right)\right\}
$$


By standard time-homogeneity arguments for infinite horizon problems we have that

$$
\begin{aligned}
e^{\beta t} V\left(t, K_{t}, X_{t}\right) & =\max _{\left\{C_{s}, M_{s}^{d}\right\}_{t \leq s<\infty}} \mathbb{E}_{t}\left[\int_{t}^{\infty} e^{-\beta(s-t)} U\left(C_{s}, M_{s}^{d}\right) d s\right] \\
& =\max _{\left\{C_{t+u}, M_{t+u}^{d}\right\}_{t \leq u<\infty}} \mathbb{E}_{t}\left[\int_{t}^{\infty} e^{-\beta u} U\left(C_{t+u}, M_{t+u}^{d}\right) d u\right] \\
& =\max _{\left\{C_{u}, M_{u}^{d}\right\}_{0 \leq u<\infty}} \mathbb{E}_{0}\left[\int_{0}^{\infty} e^{-\beta u} U\left(C_{u}, M_{u}^{d}\right) d u\right] \\
& \equiv H\left(K_{t}, X_{t}\right),
\end{aligned}
$$

where the third equality follows because the optimal robust control is Markov and $H\left(K_{t}, X_{t}\right)$ is independent of time. Therefore, we conjecture that the value function has the following form

$$
V\left(t, K_{t}, X_{t}\right)=a(t) H\left(K_{t}, X_{t}\right)=\frac{e^{-\beta t}}{\beta} H\left(K_{t}, X_{t}\right)=\frac{e^{-\beta t}}{\beta \gamma}\left(\left(e^{\phi\left(X_{t}\right)} K_{t}^{\xi}\right)^{\gamma}-1\right),
$$

where $\phi: \mathbb{R}^{2} \rightarrow \mathbb{R}$ is $\mathcal{C}^{N}$-differentiable function of the state vector $X_{t}$ that needs to be determined in equilibrium. Inserting (A.19) into the HJB equation in (A.17), the first order conditions are given by

$$
\begin{aligned}
& C_{t}^{*}= \frac{K_{t}\left(K_{t}^{Q} e^{\phi\left(X_{t}\right)}\right)^{-\gamma}}{\beta Q}\left(\frac{\left.\beta Q\left(K_{t}^{Q} e^{\phi\left(X_{t}\right)}\right)^{\gamma}\left(\left(-\frac{(\gamma-1) \beta^{\frac{1}{1-\gamma}} Q^{\frac{1}{1-\gamma}} K_{t}^{\frac{1-\gamma Q}{\gamma-1}} e^{\frac{\gamma \phi\left(X_{t}\right)}{1-\gamma}}}{\xi}\right)^{\frac{1-\gamma}{\gamma \xi+\gamma-1}}\right)^{-\xi}\right)^{\frac{\gamma}{\gamma-1}}}{K_{t}}(A .20)\right. \\
& M_{t}^{d *}=\left(\frac{(1-\gamma) \beta^{\frac{1}{1-\gamma}} Q^{\frac{1}{1-\gamma}} K_{t}^{\frac{1-\gamma Q}{\gamma-1}} e^{\frac{\gamma \phi\left(X_{t}\right)}{1-\gamma}}}{\xi}\right)^{\frac{1-\gamma}{\gamma \xi+\gamma-1}},
\end{aligned}
$$

with $Q=1+\xi$. In general, the function $\phi\left(X_{t}\right)$ cannot be obtained in closed form. However, we can obtain an asymptotic expansion to $g\left(X_{t}\right)$ with respect to the risk aversion parameter $\gamma$. Assuming a power series expression for $\phi\left(X_{t}\right)$ in $\gamma$ as follows

$$
\phi\left(X_{t}\right)=\phi_{0}\left(X_{t}\right)+\gamma \phi_{1}\left(X_{t}\right)+O\left(\gamma^{2}\right)
$$

where $\phi_{0}(X, t)$ is obtained from the logarithmic utility case, we can then solve the HJB problem in Equation (A.17) for $\gamma \neq 0$ in closed form. To do so, we solve first the HJB problem in the case where utility of the representative agent is logarithmic. 


\section{A.2.1 Log-utility case}

Note that for $\gamma \rightarrow 0$ the utility reduces to

$$
\lim _{\gamma \rightarrow 0} U\left(C_{t}, M_{t}^{d}\right)=\log \left(C_{t}\right)+\xi \log \left(M_{t}^{d}\right)
$$

The optimal consumption and investment problem is ${ }^{38}$

$$
\max _{C_{t}, M_{t}^{d}} \mathbb{E}_{0}\left[\int_{0}^{\infty} e^{-\beta t}\left[\log \left(C_{t}\right)+\xi \log \left(M_{t}^{d}\right)\right] d t\right]
$$

subject to the capital constraint in Equation (12). In equilibrium, there exists a value function $V^{\log }\left(t, K_{t}, X_{t}\right)=a(t) H^{\log }\left(K_{t}, X_{t}\right)$ and control variables $\left\{C_{t}, M_{t}^{d}\right\}$ satisfying the HJB equation

$$
-\frac{\partial\left(a(t) H^{\log }\left(K_{t}, X_{t}\right)\right)}{\partial t}=a(t)\left(\max _{\left\{C_{t}, M_{t}^{d}\right\}}\left\{U^{\log }\left(C_{t}, M_{t}^{d}\right)+\mathcal{A} H^{\log }\left(K_{t}, X_{t}\right)\right\}\right) .
$$

We consider the following linear conjecture for the value function $V^{\log (\cdot)}$

$$
a(t) H^{\log }\left(K_{t}, X_{t}\right)=\frac{e^{-\beta t}}{\beta}\left[Q \log \left(K_{t}\right)+g_{0}\left(X_{t}\right)\right],
$$

and where the function $g\left(X_{t}\right)$ is affine in the state variables, i.e.,

$$
\phi\left(X_{t}\right)=\phi_{00}+\phi_{0 A} A_{t}+\phi_{0 g} g_{t}
$$

Applying the generator to Equation (A.25), using the productivity, the government policy, and equilibrium capital accumulation dynamics in Equation (5), (6) and (20), we obtain

$$
\begin{aligned}
\mathcal{A} V^{\log } & =\frac{\partial V^{\log }}{\partial t}+\frac{\partial V^{\log }}{\partial K} \mu_{K}+\frac{\partial V^{\log }}{\partial A} \mu_{A}+\frac{\partial V^{\log }}{\partial g} \mu_{g}+\frac{1}{2} \frac{\partial^{2} V^{\log }}{\partial K^{2}} \sigma_{K}^{2} \\
& =Q\left[\mu_{Y}+q_{A} A_{t}-\left(\frac{C_{t}}{K_{t}}+\frac{M_{t}^{d}}{K_{t}}\right)-\frac{1}{2} \sigma_{Y}^{2} g_{t}\right] \\
& +\left(\kappa_{A}\left(\theta_{A}-A_{t}\right)+\lambda g_{t}\right) \phi_{0 A}+\kappa_{g}\left(\theta_{g}-g_{t}\right) \phi_{0 g} .
\end{aligned}
$$

The first order optimality conditions for consumption and money holdings are

$$
\begin{array}{r}
\frac{e^{-\beta t}}{C_{t}}-\frac{Q}{\beta} \frac{e^{-\beta t}}{K_{t}}=0 \Longleftrightarrow C_{t}^{*}=\frac{\beta K_{t}}{Q}, \\
\frac{e^{-\beta t} \xi}{M_{t}^{d}}-\frac{Q}{\beta} \frac{e^{-\beta t}}{K_{t}}=0 \Longleftrightarrow M_{t}^{d *}=\frac{\beta \xi K_{t}}{Q} .
\end{array}
$$

\footnotetext{
${ }^{38}$ The proof of this proposition is similar to the one presented in Buraschi \& Jiltsov (2005).
} 
Substituting the optimal controls $C_{t}^{*}$ and $M_{t}^{d *}$ into Equation (A.24) and matching coefficients of $\log \left(K_{t}\right), A_{t}, g_{t}$ and the constant terms, we obtain

$$
Q=1+\xi
$$

and

$$
\begin{aligned}
\phi_{00}= & \frac{(1+\xi)\left(-\theta_{g} \kappa_{g} \sigma_{Y}^{2}\left(\beta+\kappa_{A}\right)+2 \mu_{Y}\left(\beta+\kappa_{A}\right)\left(\beta+\kappa_{g}\right)+2 \theta_{g} \kappa_{g} \lambda q_{A}\right)}{2 \beta\left(\beta+\kappa_{A}\right)\left(\beta+\kappa_{g}\right)} \\
& -\frac{(1+\xi)\left(\beta(\beta+\delta)+\kappa_{A}\left(\beta+\delta-\theta_{A} q_{A}\right)\right)}{\beta\left(\beta+\kappa_{A}\right)}+L \\
\phi_{0 A}= & \frac{(1+\xi) q_{A}}{\left(\kappa_{A}+\beta\right)}, \quad \phi_{0 g}=\frac{(1+\xi)\left(\frac{2 \lambda q_{A}}{\beta+\kappa_{A}}-\sigma_{Y}^{2}\right)}{2\left(\kappa_{g}+\beta\right)}
\end{aligned}
$$

where $L$ is defined as

$$
L=\log \left(\frac{\beta^{1+\xi} \xi^{\xi}}{(1+\xi)^{1+\xi}}\right)
$$

The coefficients are all uniquely determined, state-independent and also independent of $K_{t}$. Substituting the expressions back into the HJB equations verifies that the guess was correct.

\section{A.2.2 Perturbed solution}

To derive an asymptotic approximation to the function $\phi\left(X_{t}\right)$, where the expansion taken with respect to the risk aversion parameter $\gamma$, let $V=V\left(t, K_{t}, X_{t}\right)$ denote the value function as given in Equation (A.16). Utility is now given by the following non-separable preferences

$$
U\left(C_{t}, M_{t}^{d}\right)=\frac{1}{\gamma}\left(\left(C_{t}\left(M_{t}^{d}\right)^{\xi}\right)^{\gamma}-1\right)
$$

From the HJB equation in (A.17), the optimal consumption $C_{t}^{*}$ and money demand $M_{t}^{d *}$ policy holdings are given by ${ }^{39}$

$$
\begin{gathered}
C_{t}^{*}=\left(M_{t}^{d}\right)^{-\xi}\left(\frac{Q\left(M_{t}^{d}\right)^{-\xi}\left(K_{t}^{Q} e^{\phi\left(X_{t}\right)}\right)^{\gamma}}{\beta K_{t}}\right)^{\frac{1}{\gamma-1}}, \\
M_{t}^{d *}=\left(\frac{Q C_{t}^{-\gamma} K_{t}^{\gamma Q-1} e^{\gamma \phi\left(X_{t}\right)}}{\beta \xi}\right)^{\frac{1}{\gamma \xi-1}} .
\end{gathered}
$$

\footnotetext{
${ }^{39}$ Inserting Equation (A.36) into (A.37) gives the optimal money demand in Equation (A.21) from which the optimal consumption in Equation (A.20) can easily be deduced.
} 
Inserting optimal money demand (A.37) into the first order condition of consumption (A.36), using the power series representation of $g\left(X_{t}\right)$ as given in Equation (18) and perturbing the resulting expression around the log-utility case (and analogously for optimal money demand), substituting $Q=1+\xi$ from Equation (A.31), the perturbed optimal consumption and money holdings are given by

$$
\begin{aligned}
C_{t}^{*, P} & =\frac{\beta K_{t}}{(1+\xi)}\left[1+\gamma\left(\log \left(\frac{\beta^{1+\xi} \xi^{\xi}}{(1+\xi)^{1+\xi}}\right)-\phi_{0}\left(X_{t}\right)\right)\right]+O\left(\gamma^{2}\right), \\
M_{t}^{d *, P} & =\frac{\beta \xi K_{t}}{(1+\xi)}\left[1+\gamma\left(\log \left(\frac{\beta^{1+\xi} \xi^{\xi}}{(1+\xi)^{1+\xi}}\right)-\phi_{0}\left(X_{t}\right)\right)\right]+O\left(\gamma^{2}\right) .
\end{aligned}
$$

There are a number of important conclusions that can be drawn from the optimal perturbed solutions in Equations (A.38) and (A.39). First, both equations only depend on $\phi_{0}\left(X_{t}\right)$ and do not depend on $\phi_{1}\left(X_{t}\right)$ which implies that solving the consumption-investment problem with log-utility is sufficient to fully characterize the optimal perturbed consumption and money holdings up to first order. Secondly, $C_{t}^{*, P}$ and $M_{t}^{d *, P}$ are affine functions not only of capital $K_{t}$ but also of the state vector $X_{t}$. This property of the solution will not only render the equilibrium path process of $K_{t}$ affine, but also implies that optimal inflation $d p_{t}^{*} / p_{t}^{*}$ remain affine in the state variables. Next, substituting $C_{t}^{*, P}$ and $M_{t}^{d *, P}$ into Equation (13) immediately gives the equilibrium capital process $K_{t}^{*}$ in Equation (20). To show the equilibrium price dynamics in (21), we apply Itô's lemma to the money market clearing condition $M_{t}^{S}=p_{t}^{*} M_{t}^{d *}$ and obtain ${ }^{40}$

$$
d M_{t}^{S}=M_{t}^{* d} d p_{t}^{*}+p_{t}^{*} d M_{t}^{d *}+\mathbb{C}_{t}\left(d p_{t}^{*}, d M_{t}^{* d}\right)
$$

Then using the optimal controls $C_{t}^{*}$ and $M_{t}^{d *}$ and inserting the money market clearing condition from Equation (A.40) yields

$$
\frac{d p_{t}^{*}}{p_{t}^{*}}=\frac{d M_{t}^{S}}{M_{t}^{S}}-\frac{d K_{t}^{*}}{K_{t}^{*}}-\mathbb{C}_{t}\left(\frac{d p_{t}^{*}}{p_{t}^{*}} \frac{d K_{t}^{*}}{K_{t}^{*}}\right) .
$$

Inserting the money supply rule of Equation (14) and the equilibrium capital accumulation process into (A.41) gives the equilibrium price process as in Equation (21). To verify that the guess for the value function $V(\cdot)$ was correct, we substitute the equilibrium values back into the HJB problem in Equation (A.17).

\footnotetext{
${ }^{40}$ Assuming that at $t=0$ markets are cleared. Hence, $p_{0}^{*} M_{0}^{d *}=M_{0}^{S}$.
} 


\section{A.3 Proof of Proposition 4}

To simplify notation, let $\kappa_{t}^{*}=\log \left(K_{t}^{*}\right)+\beta t$. The using the equilibrium capital accumulation process implies that $\kappa_{t}^{*}$ satisfies

$$
d \kappa_{t}^{*}=\left(\mu_{y}+q_{A} A_{t}-\delta-\frac{1}{2} \sigma_{Y}^{2} g_{t}+\beta \gamma\left(g_{0}\left(X_{t}\right)-L\right)\right) d t+\sigma_{Y} \sqrt{g_{t}} d W_{t}^{Y}
$$

The Euler condition in Equation (24) can then be expressed as

$$
\begin{aligned}
B(t, \tau) & =e^{-\beta \tau} \mathbb{E}_{t}\left[\frac{U_{C}\left(C_{t+\tau}^{*}, M_{t+\tau}^{d *}\right)}{U_{C}\left(C_{t}^{*}, M_{t}^{d *}\right)} \frac{p_{t}^{*}}{p_{t+\tau}^{*}}\right]=e^{-\beta \tau} \mathbb{E}_{t}\left[\frac{K_{t}^{*}}{K_{t+\tau}^{*}} \frac{p_{t}^{*}}{p_{t+\tau}^{*}}\right]=e^{-\beta \tau} \mathbb{E}_{t}\left[\frac{\exp \left\{-\log \left(K_{t+\tau}^{*}\right)\right\}}{\exp \left\{-\log \left(K_{t}^{*}\right)\right\}} \frac{p_{t}^{*}}{p_{t+\tau}^{*}}\right] \\
& =\mathbb{E}_{t}\left[\frac{\exp \left\{-\left(\log \left(K_{t+\tau}^{*}\right)+\beta(t+\tau)\right)\right\}}{\exp \left\{-\left(\log \left(K_{t}^{*}\right)+\beta t\right)\right\}} \frac{p_{t}^{*}}{p_{t+\tau}^{*}}\right]=\mathbb{E}_{t}\left[\frac{\exp \left\{-\kappa_{t+\tau}^{*}\right\}}{\exp \left\{-\kappa_{t}^{*}\right\}} \frac{p_{t}^{*}}{p_{t+\tau}^{*}}\right] .
\end{aligned}
$$

To solve the problem in Equation (A.43) we follow Ulrich (2013) and Buraschi \& Jiltsov (2005) and define

$$
f=f\left(\kappa_{t}^{*}, p_{t}^{*}, A_{t}, g_{t}, m_{t}, \tau\right)=\mathbb{E}_{t}\left[\frac{e^{-\kappa_{t+\tau}^{*}}}{p_{t+\tau}^{*}}\right]
$$

Then, conjecturing a log-linear guess for $f(\cdot)$ of the form

$$
f\left(\kappa_{t}^{*}, p_{t}^{*}, A_{t}, g_{t}, m_{t}, \tau\right)=\frac{e^{-\kappa_{t}^{*}}}{p_{t}^{*}} \exp \left\{-b_{0}(\tau)-b_{A}(\tau) A_{t}-b_{g}(\tau) g_{t}-b_{m}(\tau) m_{t}\right\} .
$$

If our log-linear guess in Equation (A.45) solves the stochastic problem in (A.44) then it is also the solution to the following PDE

$$
-\frac{\partial f(\cdot, \tau)}{\partial \tau}=\mathcal{A} f(\cdot, \tau), f(\cdot, 0)=\frac{e^{-\kappa_{t}^{*}}}{p_{t}^{*}}
$$

The left-hand side of Equation (A.46) is given by

$$
\frac{\partial f(\cdot, \tau)}{\partial \tau}=\left[-b_{0}^{\prime}(\tau)-b_{A}^{\prime}(\tau) A_{t}-b_{g}^{\prime}(\tau) g_{t}-b_{m}^{\prime}(\tau) m_{t}\right] f(\cdot, \tau)
$$

Setting $\Theta=\left\{\kappa_{t}^{*}, p_{t}^{*}, A_{t}, g_{t}, m_{t}\right\}$, an application of Itô's lemma to the right-hand side of (A.46) gives

$$
\mathcal{A} f\left(\kappa_{t}^{*}, p_{t}^{*}, A_{t}, g_{t}, m_{t}, \tau\right)=\sum_{i \in \Theta} \frac{\partial f}{\partial \Theta^{i}} \mu_{\Theta^{i}} d t+\frac{1}{2} \sum_{i \in \Theta} \frac{\partial^{2} f}{\partial \widetilde{\Theta}^{i 2}} d\left\langle\Theta^{i}, \Theta^{i}\right\rangle_{t}+\sum_{\substack{i, j \in \Theta \\ i \neq j}} \frac{\partial^{2} f}{\partial \Theta^{i} \Theta^{j}} d\left\langle\Theta^{i}, \Theta^{j}\right\rangle_{t} .
$$


Writing out the expression above, recalling that $\rho^{g m}=\rho^{A g}=\rho^{M A}=\rho^{M g}=0$ and substituting the dynamics of $\kappa_{t}^{*}, p_{t}^{*}, A_{t}, g_{t}$, and $m_{t}$, we get

$$
\begin{aligned}
\mathcal{A f} & =\frac{\partial f}{\partial \kappa^{*}}\left(\mu_{K^{*}}\left(A_{t}, g_{t}\right)-\frac{1}{2} \sigma_{Y}^{2} g_{t}\right) \\
& +\frac{\partial f}{\partial p_{t}^{*}} p_{t}^{*}\left[\frac{\mu_{M}-\eta_{1} \bar{k}-\eta_{2} \bar{\pi}}{1-\eta_{2}}+\frac{\eta_{1}-1}{1-\eta_{2}} \mu_{K^{*}}\left(A_{t}, g_{t}\right)-g_{t} \frac{\left(\eta_{1}-1\right) \sigma_{Y}^{2}}{1-\eta_{2}}\right] \\
& +\frac{\partial f}{\partial A}\left(\kappa_{A}\left(\theta_{A}-A_{t}\right)+\lambda g_{t}\right)+\frac{\partial f}{\partial g} \kappa_{g}\left(\theta_{g}-g_{t}\right)+\frac{\partial f}{\partial m} \kappa_{m}\left(\theta_{m}-m_{t}\right) \\
& +\frac{1}{2}\left[\frac{\partial^{2} f}{\partial \kappa^{* 2}} \sigma_{Y}^{2} g_{t}+\frac{\partial^{2} f}{\partial p^{* 2}} p_{t}^{* 2}\left[g_{t}\left(\frac{\eta_{1}-1}{1-\eta_{2}}\right)^{2} \sigma_{Y}^{2}+m_{t} \frac{\sigma_{M}^{2}}{\left(1-\eta_{2}\right)^{2}}\right]\right. \\
& \left.+\frac{\partial^{2} f}{\partial A^{2}} \sigma_{A}^{2} g_{t}+\frac{\partial^{2} f}{\partial g^{2}} \sigma_{g}^{2} g_{t}+\frac{\partial^{2} f}{\partial m^{2}} \sigma_{m}^{2} m_{t}\right] \\
& +\frac{\partial^{2} f}{\partial \kappa^{*} \partial p^{*}} p_{t}^{*} \sigma_{Y}^{2} g_{t} \frac{\eta_{2}-1}{1-\eta_{2}}+\frac{\partial^{2} f}{\partial \kappa^{*} \partial A} \sigma_{A} \sigma_{Y} \rho^{A Y} g_{t}+\frac{\partial^{2} f}{\partial \kappa^{*} \partial g} \sigma_{g} \sigma_{Y} \rho^{g Y} g_{t} \\
& +\frac{\partial^{2} f}{\partial p^{*} \partial A} p_{t}^{*} \frac{\eta_{1}-1}{1-\eta_{2}} \sigma_{A} \sigma_{Y} g_{t} \rho^{A Y}+\frac{\partial^{2} f}{\partial p^{*} \partial g} p_{t}^{*} \frac{\eta_{1}-1}{1-\eta_{2}} \sigma_{g} \sigma_{Y} g_{t} \rho^{g Y} .
\end{aligned}
$$

Computing the derivatives and separating variables one obtains the following system of first order asymptotic (Riccati) ODE's:

$$
\begin{aligned}
0 & =-C_{A}+\kappa_{A} b_{A}(\tau)+b_{A}^{\prime}(\tau), \\
0 & =Z_{0 g}(\tau)+b_{g}(\tau) Z_{1 g}(\tau)+Z_{2 g} b_{g}^{2}(\tau)+b_{g}^{\prime}(\tau), \\
0 & =Z_{0 m}+b_{m}(\tau) Z_{1 m}+Z_{2 m} b_{m}^{2}(\tau)+b_{m}^{\prime}(\tau), \\
0 & =-b_{0}^{\prime}(\tau)+C_{0}(\tau)
\end{aligned}
$$


subject to $b_{A}(0)=b_{g}(0)=b_{m}(0)=b_{0}(0)=0$ and where

$$
\begin{aligned}
C_{A} & =\left(q_{A}+\gamma \beta \phi_{0 A}\right)\left(\frac{\eta_{1}-\eta_{2}}{1-\eta_{2}}\right) \\
Z_{0 g}(\tau) & =\left(q_{A}^{2}+2 \gamma \beta \phi_{0 A}\right)\left(\frac{\eta_{1}-\eta_{2}}{1-\eta_{2}}\right)^{2} \frac{\left(1-e^{-\kappa_{A} \tau}\right)^{2} \sigma_{A}^{2}}{2 \kappa_{A}^{2}}+\frac{\left(\eta_{1}-\eta_{2}\right)^{2} \sigma_{Y}^{2}}{\left(\eta_{2}-1\right)^{2}}+\gamma \frac{\beta \phi_{0 g}\left(\eta_{1}-\eta_{2}\right)}{\eta_{2}-1} \\
& -b_{A}(\tau)\left(\frac{\left(\eta_{2}-1\right) \lambda+\left(\eta_{1}-\eta_{2}\right) \rho^{A Y} \sigma_{A} \sigma_{Y}}{\eta_{2}-1}\right), \\
Z_{1 g}(\tau) & =\kappa_{g}+b_{A}(\tau) \rho^{A g} \sigma_{A} \sigma_{g}+\frac{\left(\eta_{2}-\eta_{1}\right) \rho^{g Y} \sigma_{g} \sigma_{Y}}{\eta_{2}-1}, \\
Z_{2 g} & =\sigma_{g}^{2} / 2, H_{g}(\tau)=\sqrt{4 Z_{0 g}(\tau) Z_{2 g}-Z_{1 g}^{2}}, \\
Z_{0 m} & =\frac{\sigma_{M}^{2}}{\left(\eta_{2}-1\right)^{2}}, Z_{1 m}=\kappa_{m}+\frac{\rho^{M m} \sigma_{m} \sigma_{M}}{1-\eta_{2}}, Z_{2 m}=\frac{\sigma_{m}^{2}}{2}, H_{m}=\sqrt{4 Z_{0 m} Z_{2 m}-Z_{1 m}^{2}} \\
C_{0}(\tau) & =\frac{\left(\mu_{Y}-\beta-\delta-\bar{k}\right) \eta_{1}+\beta+\mu_{M}-\eta_{2}\left(\mu_{Y}+\bar{\pi}-\delta\right)}{1-\eta_{2}} \\
& +\sum_{i \in\{A, g, m\}} b_{i}(\tau) \theta_{i} \kappa_{i}+\gamma \frac{\beta\left(\eta_{1}-\eta_{2}\right)\left(L-\phi_{00}\right)}{\eta_{2}-1} .
\end{aligned}
$$

For the existence of a solution to the bond pricing PDE that excludes arbitrage opportunities, requires that the Riccati equations in (A.50) and (A.51) above, satisfy the following periodicity condition

$$
4 Z_{0 g}(\tau) Z_{2 g}-Z_{1 g}^{2}(\tau)<0,4 Z_{0 m} Z_{2 m}-Z_{1 m}^{2}<0, \forall \tau \geq 0
$$

This condition essentially rules out singularities of the solution of the Riccati equation above, i.e., for $\tau \geq 0$, the function $b_{i}(\tau), i \in\{g, m\}$ is continuous in $\tau$.

\section{A.4 Proof of Proposition 5}

The unconditional correlation coefficient of the nominal yield curve $Y(t, \tau)$ and $g_{t}$ is given by

$$
\varrho\left[Y(t, \tau), g_{t}\right]=\frac{\mathbb{C}\left[Y(t, \tau), g_{t}\right]}{\sqrt{\mathbb{V}[Y(t, \tau)] \mathbb{V}\left[g_{t}\right]}}
$$

Using the affine expression in Equation (30) and the results from Proposition 1, the unconditional covariance is

$$
\mathbb{C}\left[Y(t, \tau), g_{t}\right]=\frac{b_{A}(\tau)}{\tau} \mathbb{C}\left[A_{t}, g_{t}\right]+\frac{b_{g}(\tau)}{\tau} \mathbb{V}\left[g_{t}\right]=\frac{b_{A}(\tau)}{\tau} \frac{\theta_{g} \sigma_{g}\left(2 \kappa_{g} \lambda \rho^{A g} \sigma_{A}+\lambda \sigma_{g}\right)}{2 \kappa_{g}\left(\kappa_{A}+\kappa_{g}\right)}+\frac{b_{g}(\tau)}{\tau} \frac{\theta_{g} \sigma_{g}^{2}}{2 \kappa_{g}},
$$


where the unconditional variance of term structure is $\mathbb{V}[Y(t, \tau)]=\frac{b_{A}^{2}(\tau)}{\tau^{2}} \mathbb{V}\left[A_{t}\right]+\frac{b_{g}^{2}(\tau)}{\tau^{2}} \mathbb{V}\left[g_{t}\right]+\frac{b_{m}^{2}(\tau)}{\tau^{2}} \mathbb{V}\left[m_{t}\right]+$ $2 \frac{b_{A}(\tau)}{\tau} \frac{b_{g}(\tau)}{\tau} \mathbb{C}\left[A_{t}, g_{t}\right]$. Along the same line of argumentation, we have that

$$
\mathbb{C}\left(Y(t, \tau), m_{t}\right)=\frac{\theta_{m} \sigma_{m}^{2}}{2 \kappa_{m}} \frac{b_{m}(\tau)}{\tau}
$$

and since $b_{m}(\tau) \leq 0, \tau \geq 0$ the result follows immediately.

\section{A.5 Proof of Proposition 6}

In this section we derive the first order asymptotic nominal short rate and the market prices of real and nominal risks when the investor has CRRA utility. The nominal short rate is defined as the following limit

$$
R_{t}:=\lim _{\tau \rightarrow 0} Y(t, \tau)=\lim _{\tau \rightarrow 0}-\frac{1}{\tau}(\log (B(t, \tau)))
$$

To prove Equation (34) we make repeated use of Bernoulli's rule. For instance, to compute $\lim _{\tau \rightarrow 0} \frac{b_{0}(\tau)}{\tau}$ we set $f(\tau):=\int_{0}^{\tau} C_{0}(u) d u$ and $g(\tau)=\tau$. Then, using Leibniz' integral rule we obtain

$$
\begin{aligned}
\lim _{\tau \rightarrow 0} \frac{f(\tau)}{g(\tau)} & =\lim _{\tau \rightarrow 0} \frac{f^{\prime}(\tau)}{g^{\prime}(\tau)} \\
& =\lim _{\tau \rightarrow 0} C_{0}(\tau)=\frac{\left(\mu_{Y}-\beta-\delta-\bar{k}\right) \eta_{1}+\beta+\mu_{M}-\eta_{2}\left(\mu_{Y}+\bar{\pi}-\delta\right)}{1-\eta_{2}}+\gamma \frac{\beta\left(\eta_{1}-\eta_{2}\right)\left(L-\phi_{00}\right)}{\eta_{2}-1},
\end{aligned}
$$

Similarly we have

$$
\begin{aligned}
& \lim _{\tau \rightarrow 0} \frac{b_{A}(\tau)}{\tau}=C_{A}, \\
& \lim _{\tau \rightarrow 0} \frac{b_{g}(\tau)}{\tau}=-Z_{0 g}(0)=-\left(\frac{\left(\eta_{1}-\eta_{2}\right)^{2} \sigma_{Y}^{2}}{\left(\eta_{2}-1\right)^{2}}+\gamma \frac{\beta \phi_{0 g}\left(\eta_{1}-\eta_{2}\right)}{\eta_{2}-1}\right), \\
& \lim _{\tau \rightarrow 0} \frac{b_{m}(\tau)}{\tau}=-Z_{0 m}=-\frac{\sigma_{M}^{2}}{\left(\eta_{2}-1\right)^{2}}
\end{aligned}
$$

which gives the expression for the short rate in Equation (34). Next, in order to derive the bond excess return, we apply Itô's rule to the closed-form bond price formula in Equation (25) and obtain the following dynamics

$$
\frac{d B(t, \tau)}{B(t, \tau)}=\frac{\partial B(t, \tau)}{\partial t} d t+\sum_{i \in \Theta} \frac{\partial B(t, \tau)}{\partial \Theta^{i}} d \Theta_{t}^{i}+\frac{1}{2} \sum_{i \in \Theta} \frac{\partial^{2} B(t, \tau)}{\partial \Theta^{i 2}} d\left\langle\Theta^{i}, \Theta^{i}\right\rangle_{t},
$$


where $\Theta=\{A, g, m\}$. Taking time $t$ conditional expectation on both sides and using Equation (A.50) and (A.51) from above, we obtain that the expected infinitesimal bond risk premia is given by $R P(t, \tau):=\frac{1}{d t} \mathbb{E}_{t}\left[\frac{d B(t, \tau)}{B(t, \tau)}-R_{t} d t\right]=\frac{\eta_{2}-\eta_{1}}{\eta_{2}-1} \sigma_{Y}\left[b_{A}(\tau) \rho^{A Y} \sigma_{A}+\sigma_{g} b_{g}(\tau) \rho^{g Y}\right] g_{t}+b_{m}(\tau) \frac{\sigma_{M} \sigma_{m} \rho^{M m}}{\eta_{2}-1} m_{t}$,

which is after defining the nominal market price of risks the expression in Equation (37). The results for the log-utility agent can be easily derived by simply setting $\gamma=0$ in Proposition (6). However, for verifying our results and to explain why we defined the market prices of government $\lambda_{t}^{N, Y}$ and monetary policy risk $\lambda_{t}^{N, M}$ as above, we derive both the nominal short rate, the market prices of risk and the term premium from a different angle using the stochastic discount factor approach. Recall that the real short rate can be obtained directly from the real stochastic discount factor which we denote by $\zeta_{t}^{R}$. Its dynamics take the general form

$$
d \zeta_{t}^{R}=\mu\left(\zeta^{R}, t\right) d t+\boldsymbol{\sigma}\left(\zeta^{R}, t\right)^{\prime} d \boldsymbol{W}_{t}
$$

where $\mu(\cdot, \cdot)$ and $\boldsymbol{\sigma}(\cdot, \cdot)$ are $\mathcal{F}_{t}$-measurable bounded scalar drift and vector diffusion processes and $\boldsymbol{W}_{t}=\left(W_{t}^{Y}, W_{t}^{M}\right)$. In our setup, $\zeta_{t}^{R}=e^{-\rho t} U_{C}\left(X_{t}^{*}\right)$ from which, after an application of Itô's lemma we find

$$
\frac{d \zeta_{t}^{R}}{\zeta_{t}^{R}}=-\left(\mu_{Y}+q_{A} A_{t}-\delta-\sigma_{Y}^{2} g_{t}\right) d t-\sigma_{y} \sqrt{g_{t}} d W_{t}^{Y}
$$

from which we can read off the real short rate as $r_{t}=-\mu\left(\zeta^{R}, t\right)$ and the market price of the real productivity innovation risk by $\lambda_{t}^{R, \cdot}=\sigma_{Y} \sqrt{g}_{t}$. Following Veronesi \& Jared (2000) or Piazzesi \& Schneider (2006), the dynamics of the nominal stochastic discount factor $\zeta_{t}^{N}$ are given by

$$
\begin{aligned}
-\frac{d \zeta_{t}^{N}}{\zeta_{t}^{N}} & =\left\{\frac{\mu_{M}+\beta\left(1-\eta_{1}\right)-\eta_{1} \bar{k}-\eta_{2} \bar{\pi}-\left(\eta_{1}-\eta_{2}\right)\left(\delta-\mu_{Y}\right)}{\eta_{2}-1}+\frac{q_{A}\left(\eta_{1}-\eta_{2}\right)}{1-\eta_{2}} A_{t}\right. \\
& \left.-\frac{\left(\eta_{1}-\eta_{2}\right)^{2} \sigma_{Y}^{2}}{\left(\eta_{2}-1\right)^{2}} g_{t}-\frac{\sigma_{M}^{2}}{\left(\eta_{2}-1\right)^{2}} m_{t}\right\} d t+\frac{\left(\eta_{1}-\eta_{2}\right) \sigma_{Y}}{1-\eta_{2}} \sqrt{g_{t}} d W_{t}^{Y}+\frac{\sigma_{M}}{1-\eta_{2}} \sqrt{m_{t}} d W_{t}^{M}
\end{aligned}
$$

from which we deduce that $R_{t}=-\mu\left(\zeta^{N}, t\right)$. Similarly we have that the nominal market prices of risk is given by $\lambda_{t}^{N, \cdot}=-\boldsymbol{\sigma}\left(\zeta^{N}, \boldsymbol{t}\right)$. Next, in order to determine the term premium on nominal bond yields under log-utility, we make use of the closed-form solution of the nominal bond price as given in Proposition 4, and the nominal short rate together with the associated market prices $\lambda_{t}^{N, Y}$ and $\lambda_{t}^{N, M}$ as in Proposition 6. An application of Itô's lemma to Equation (25) shows that the risk-neutral 
dynamics of the bond price equals to

$$
\frac{d B(t, \tau)}{B(t, \tau)}=R_{t} d t-b_{A}(\tau) \sigma_{A} \sqrt{g_{t}} d \widetilde{W}_{t}^{A}-b_{g}(\tau) \sigma_{g} \sqrt{g_{t}} d \widetilde{W}_{t}^{g}-b_{m}(\tau) \sigma_{m} \sqrt{m_{t}} d \widetilde{W}_{t}^{m}
$$

where $\widetilde{W}_{t}^{i}, i \in\{A, g, m\}$ are $\mathcal{F}_{t}$-measurable Brownian innovation processes under the risk neutral measure $\mathbb{Q}$. According to Proposition 4, only government and nominal monetary policy risk are priced. We can decompose the Brownian innovations $\widetilde{W}_{t}^{A}, \widetilde{W}_{t}^{g}$ and $\widetilde{W}_{t}^{m}$ into two orthogonal complements as follows

$$
\begin{array}{rlrl}
d \widetilde{W}_{t}^{A} & =\rho^{A Y} d \widetilde{W}_{t}^{Y}+\sqrt{1-\rho^{A Y}} d \widehat{W}_{t}^{A}, & d \widetilde{W}_{t}^{Y} d \widehat{W}_{t}^{A}=0, \\
d \widetilde{W}_{t}^{g}=\rho^{Y g} d \widetilde{W}_{t}^{Y}+\sqrt{1-\rho^{Y g^{2}}} d \widehat{W}_{t}^{g}, & d \widetilde{W}_{t}^{Y} d \widehat{W}_{t}^{g}=0 \\
d \widetilde{W}_{t}^{m}=\rho^{M m} d \widetilde{W_{t}^{M}}+\sqrt{1-\rho^{M m^{2}}} d \widehat{W}_{t}^{m}, & d \widehat{W}_{t}^{M} d \widehat{W}_{t}^{Y}=0 .
\end{array}
$$

Hence, substituting the decomposed Brownian motions into Equation (A.68), the bond price dynamics are then given by

$$
\begin{aligned}
\frac{d B(t, \tau)}{B(t, \tau)} & =R_{t} d t-b_{A}(\tau) \sigma_{A} \sqrt{g_{t}}\left(\rho^{A Y} d \widetilde{W}_{t}^{Y}+\sqrt{1-\rho^{A Y}} d \widehat{W}_{t}^{A}\right) \\
& -b_{g}(\tau) \sigma_{g} \sqrt{g_{t}}\left(\rho^{Y g} d \widetilde{W}_{t}^{Y}+\sqrt{1-\rho^{Y g^{2}}} d \widehat{W}_{t}^{g}\right) \\
& -b_{m}(\tau) \sigma_{m} \sqrt{m_{t}}\left(\rho^{M m} d \widetilde{W}_{t}^{M}+\sqrt{1-\rho^{M m^{2}}} d \widehat{W}_{t}^{m}\right) .
\end{aligned}
$$

Next, in order to express the bond under the physical measure $\mathbb{P}$ we apply Girsanov's theorem to perform a measure change from $\mathbb{Q}$ to $\mathbb{P}$ as follows. We set the change of the drift equal to the market price of risk, in other words we have $d \widetilde{W}_{t}^{Y}=\lambda_{t}^{N, Y} d t+d W_{t}^{Y}$ and $d \widetilde{W}_{t}^{M}=\lambda_{t}^{N, M} d t+d W_{t}^{M}$. Then, given the correlation structure of the factors we obtain that the equilibrium term premium under the physical measure $\mathbb{P}$ is given by

$$
\frac{1}{d t} \mathbb{E}_{t}\left[\frac{d B(t, \tau)}{B(t, \tau)}-R_{t} d t\right]=\lambda_{t}^{N, Y}\left[b_{A}(\tau) \rho^{A Y} \sigma_{A}+\sigma_{g} b_{g}(\tau) \rho^{g Y}\right] \sqrt{g_{t}}-b_{m}(\tau) \lambda_{t}^{N, M} \sqrt{m_{t}} .
$$




\section{B Supplementary Tables}

\begin{tabular}{cccccccccc}
\hline & EPU & GPU & MPU & $1 \mathrm{Y}$ & $2 \mathrm{Y}$ & $3 \mathrm{Y}$ & $5 \mathrm{Y}$ & $7 \mathrm{Y}$ & $10 \mathrm{Y}$ \\
\hline EPU & 1 & 0.857 & 0.657 & -0.544 & -0.542 & -0.533 & -0.500 & -0.468 & -0.425 \\
GPU & & 1 & 0.572 & -0.450 & -0.444 & -0.435 & -0.401 & -0.369 & -0.324 \\
MPU & & & 1 & -0.036 & -0.037 & -0.026 & 0.006 & 0.029 & 0.056 \\
1Y & & & & 1 & 0.993 & 0.982 & 0.952 & 0.927 & 0.896 \\
$2 \mathrm{Y}$ & & & & & 1 & 0.997 & 0.979 & 0.961 & 0.936 \\
$3 \mathrm{Y}$ & & & & & & 1 & 0.991 & 0.979 & 0.958 \\
$5 \mathrm{Y}$ & & & & & & & 1 & 0.997 & 0.987 \\
$7 \mathrm{Y}$ & & & & & & & & 1 & 0.996 \\
$10 \mathrm{Y}$ & & & & & & & & & 1.000 \\
\hline
\end{tabular}

Table 6: Sample correlation matrix of EPU, GPU and MPU index with nominal yields with $\tau=1 \mathrm{Y}, 2 \mathrm{Y}, 3 \mathrm{Y}$, 5Y, 7Y and 10Y using data from January 1990 until June 2014.

\begin{tabular}{lrrrrrrrrr}
\hline & EPU & GPU & \multicolumn{1}{c}{ MPU } & \multicolumn{1}{c}{$1 \mathrm{Y}$} & \multicolumn{1}{c}{$2 \mathrm{Y}$} & \multicolumn{1}{c}{$3 \mathrm{Y}$} & \multicolumn{1}{c}{$5 \mathrm{Y}$} & \multicolumn{1}{c}{$7 \mathrm{Y}$} & \multicolumn{1}{c}{$10 \mathrm{Y}$} \\
\hline EPU & 1 & 0.8573 & 0.6573 & 0.6272 & 0.6611 & 0.664 & 0.6533 & 0.6254 & 0.6048 \\
GPU & & 1 & 0.5724 & 0.4898 & 0.527 & 0.5211 & 0.5218 & 0.495 & 0.462 \\
MPU & & & 1 & 0.1081 & 0.1236 & 0.1451 & 0.1309 & 0.1175 & 0.1243 \\
$1 Y$ & & & & 1 & 0.9406 & 0.9214 & 0.8784 & 0.8497 & 0.8261 \\
$2 \mathrm{Y}$ & & & & & 1 & 0.9887 & 0.9644 & 0.9381 & 0.9067 \\
$3 \mathrm{Y}$ & & & & & & 1 & 0.9815 & 0.9595 & 0.9335 \\
$5 \mathrm{Y}$ & & & & & & & 1 & 0.9935 & 0.9728 \\
$7 \mathrm{Y}$ & & & & & & & & 1 & 0.9877 \\
$10 \mathrm{Y}$ & & & & & & & & & 1 \\
\hline
\end{tabular}

Table 7: Sample correlation matrix of EPU, GPU and MPU index with realized volatility as in Equation (41) with $\tau=1 \mathrm{Y}, 2 \mathrm{Y}, 3 \mathrm{Y}, 5 \mathrm{Y}, 7 \mathrm{Y}$ and 10Y using data from January 1990 until June 2014.

\begin{tabular}{cccccccc}
\hline & $\tau$ & $1 \mathrm{Y}$ & $2 \mathrm{Y}$ & $3 \mathrm{Y}$ & $5 \mathrm{Y}$ & $7 \mathrm{Y}$ & $10 \mathrm{Y}$ \\
\hline \multirow{2}{*}{ Full regression } & GPU & 0.051 & 0.0558 & $0.05^{*}$ & $0.042^{* *}$ & $0.032^{*}$ & $0.022^{* *}$ \\
& $t_{G P U}$ & $(2.89)$ & $(5.36)$ & $(5.37)$ & $(8.22)$ & $(8.03)$ & $(7.46)$ \\
& $R_{a d j}^{2}$ & 0.69 & 0.81 & 0.80 & 0.83 & 0.83 & 0.83 \\
\hline \multirow{2}{*}{ Full Regression } & $\mathrm{MPU}$ & 0.011 & -0.012 & $0.011^{*}$ & $0.01^{* *}$ & $0.007^{*}$ & $0.05^{* *}$ \\
& $t_{M P U}$ & $(1.11)$ & $(1.49)$ & $(1.67)$ & $(1.961)$ & $(1.91)$ & $(1.961)$ \\
& $R_{a d j}^{2}$ & 0.65 & 0.76 & 0.75 & 0.76 & 0.76 & 0.77 \\
\hline
\end{tabular}

Table 8: Summary of regression results: Table displays the slope coefficients of the regression of $\mathcal{V}_{t}[Y(t, \tau)]$ on $M P U_{t}$ and $G P U_{t}$ individually, plus the economic condition controls (EC), the financial variables (FV) plus macro factors (RA and IF) for $\tau=1 \mathrm{Y}, 2 \mathrm{Y}, 3 \mathrm{Y}, 5 \mathrm{Y}, 7 \mathrm{Y}$, and $10 \mathrm{Y}$. Values in brackets below represent HAC-robust $t$-statistics. $R_{a d j}^{2}$ refers to adjusted coefficient of determination. By ***, **, * we denote $1 \%$, $5 \%$, and $10 \%$ statistical significance, respectively. 


\section{References}

Ang, A. \& Piazzesi, M. (2003), 'A no-arbitrage vector autoregression of term structure dynamics with macroeconomic and latent variables', Journal of Monetary Economics 50, 745-787.

Baker, S., Bloom, N. \& Davis, S. J. (2012), 'Measuring economic policy uncertainty', Manuscript Stanford University .

Balduzzi, P., Elton, E. \& Green, T. (2001), 'Economic news and bond prices: evidence from the u.s. treasury market.', Journal of Financial and Quantitative Analysis 36, 523-543.

Bansal, R. \& Shaliastovich, I. (2013), 'A Long-Run Risks Explanation of Predictability Puzzles in Bond and Currency Markets', Review of Financial Studies 26(1), 1-33.

URL: http://ideas.repec.org/a/oup/rfinst/v26y2013i1p1-33.html

Bansal, R. \& Yaron, A. (2004), 'Risks for the long run: A potential resolution of asset pricing puzzles', The Journal of Finance 59, 1481-1509.

Bauer, M. D. \& Rudebusch, G. D. (2015), 'Resolving the spanning puzzle in macro-finance term structure models'.

Bekaert, G., Engstrom, E. \& Xing, Y. (2009), 'Risk, uncertainty, and asset prices', Journal of Financial Economics 91(1), 59-82.

Bekaert, G., Harvey, C. R., Lundblad, C. T. \& Siegel, S. (2014), 'Political risk spreads', Journal of International Business Studies 45(4), 471-493.

Belo, F., Gala, V. \& Li, J. (2013), 'Government spending, political cycles and the cross section of stock returns', Journal of Financial Economics 107, 305-324.

Bialkowski, J., Gottschalk, K. \& Wisnieski, T. (2008), 'Stock market volatility around national elections', Journal of Banking and Finance 32, 1941-1953.

Bloom, N. (2009), 'The impact of uncertainty shocks', Econometrica 77(3), 623-685.

Bond, P. \& Goldstein, I. (2012), 'Government intervention and information aggregation by prices', Working Paper . 
Boutchkova, M. K., Doshi, H., Durnev, A. \& Molchanov, A. (2012), 'Precarious politics and return volatility', The Review of Financial Studies 25, 1111-1154.

Brogaard, J. \& Detzel, A. (2012), 'The asset pricing implications of government economic policy uncertainty', Working Paper .

Buraschi, A., Carnelli, A. \& Whelan, P. (2014), 'Monetary policy and treasury risk premia', Working Paper .

Buraschi, A. \& Jiltsov, A. (2005), 'Inflation risk premia and the expectation hypothesis', Journal of Financial Economics 75, 429-490.

Buraschi, A. \& Jiltsov, A. (2007), 'Habit formation and macroeconomic models of the term structure of interest rates', The Journal of Finance 6, 3009-30063.

Campbell, J. Y. \& Shiller, R. J. (1991), 'Yield spreads and interest rate movements: A bird's eye view', Review of Economic Studies 58, 495-514.

Cochrane, J. \& Piazzesi, M. (2005), 'Bond risk premia', American Economic Review 95, 138-160.

Cox, J., Iingersoll, J. \& Ross, S. (1985), 'A theory of the term structure of interest rates', Econometrica 53, 385-408.

David, A. \& Veronesi, P. (2014), 'Investors' and central bank's uncertainty embedded in index options', Review of Financial Studies 27(6), 1661-1716.

de Goeij, P. \& Marquering, W. (2006), 'Macroeconomic announcements and asymmetric volatility in bond returns', Journal of Banking and Finance 30(10), 2659-2680.

Duffie, D. \& Kan, R. (1996), 'A yield factor model of interest rates', Mathematical Finance 6, 379406.

Durnev, A. (2010), 'The real effects of political uncertainty: Elections and investment sensitivity to stock prices', Working Paper University of Iowa.

Evans, C. \& Marshall, D. (2007), 'Economic determinants of the nominal treasury yield curve', Journal of Monetary Economics 54, 1986-2003. 
Fama, E. \& French, K. (1989), 'Business conditions and expected returns on stocks and bonds', Journal of Financial Economics 25, 23-49.

Fisher, S. \& Modigliani, F. (1978), 'Towards an understanding of the real effects and costs of inflation', Weltwirtschaftliches Archiv 114, 810-832.

Fleming, M. \& Piazzesi, M. (2005), 'Monetary policy tick-by-tick', Working Paper .

Gallmeyer, M. F., Hollifield, B., Palomino, F. \& Zin, S. E. (2007), Arbitrage-free bond pricing with dynamic macroeconomic models, Working Paper 13245, National Bureau of Economic Research. URL: http://www.nber.org/papers/w13245

Gürkaynak, R. S., Sack, B. \& Swanson, E. (2005a), 'Do actions speak louder than words? the response of asset prices to monetary policy actions and statements', International Journal of Central Banking pp. 55-93.

Gürkaynak, R. S., Sack, B. \& Swanson, E. (2005b), 'The sensitivity of long-term interest rates to economic news: Evidence and implications for macroeconomic models', The American Economic Review 95, 425-426.

Gulen, H. \& Ion, M. (2012), 'Policy uncertainty and corporate investment', Working Paper .

Hansen, L. P., Heaton, J. C. \& Li, N. (2008), 'Consumption strikes back? measuring long?run risk', Journal of Political Economy 116(2), pp. 260-302.

Huang, T., Wu, F., Yu, J. \& Zhang, B. (2015), 'International political risk and government bond pricing', Journal of Banking and Finance 55, 393-405.

Joslin, S., Priebisch, M. \& Singleton, K. (2014), 'Risk premiums in dynamic term structure models with unspanned macro risks', The Journal of Finance 69, 1197-1233.

Joslin, S., Priebsch, M. \& Singleton, K. J. (2014), 'Risk Premiums in Dynamic Term Structure Models with Unspanned Macro Risks', Journal of Finance 69(3), 1197-1233.

URL: http://ideas.repec.org/a/bla/jfinan/v69y2014i3p1197-1233.html 
Julio, J. \& Yook, Y. (2012), 'Political uncertainty and corporate investment cycles', Journal of Finance 67, 45-83.

Kelly, B., Pastor, L. \& Veronesi, P. (2013), 'The price of political uncertainty: Theory and evidence from the option market', Working Paper .

Kogan, L. \& Uppal, R. (2001), Risk aversion and optimal portfolio policies in partial and general equilibrium economies, Working paper, Sloan School of Management, MIT.

Kuttner, K. N. (2001), 'Monetary policy surprises and interest rates: Evidence from the fed funds futures market', Journal of Monetary Economics 47, 523-544.

Litterman, R. B. \& Scheinkman, J. (1991), 'Common factors affecting bond returns', The Journal of Fixed Income 1, 54-61.

Ludvigson, S. \& Ng, S. (2009), 'Macro factors in bond risk premia', Review of Financial Studies 22, 5027-5067.

Newey, W. \& West, K. (1994), 'Automatic lag selection in covariance estimation', Review of Economic Studies 61, 631-653.

Øksendal, B. (2003), Stochastic differential equations, Springer.

Pastor, L. \& Veronesi, P. (2012), 'Uncertainty about government policy and stock prices', Journal of Finance 4, 1219-1264.

Pastor, L. \& Veronesi, P. (2013), 'Political uncertainty and risk premia', Journal of Financial Economics 110, 520-545.

Pennacchi, G. (1991), 'Identifying the dynamics of real interest rates and inflation evidence using survey data', The Review of Financial Studies 4, 53-86.

Piazzesi, M. (2005), 'Bond yields and the federal reserve', Journal of Political Economy 112, 311-344.

Piazzesi, M. \& Schneider, M. (2006), 'Equilibrium yield curves', NBER Working Paper Series . 
Pindyck, R. \& Solimano, A. (1993), 'Economic instability and aggregate investment', NBER Working Paper Series .

Rodrik, D. (1991), 'Policy uncertainty and private investment in developing countries', Journal of Development Economics 36, 229-242.

Shiller, R. (1979), 'The volatility of long-term interest rates and expectations models of the term structure', Journal of Political Economy 87, 1190-1219.

Ulrich, M. (2013), 'Inflation ambiguity and the term structure of u.s. government bonds', Journal of Monetary Economics 60, 295-309.

Vasicek, O. (1977), 'An equilibrium characterization of the term structure', Journal of Financial Economics 5, 177-188.

Veronesi, P. \& Jared, F. (2000), 'Short and long horizon term and inflation risk premia in the us term structure', Working Paper.

Wachter, J. A. (2006), 'A consumption-based model of the term structure of interest rates', Journal of Financial economics 79(2), 365-399.

Wright, J. (2012), 'What does monetary policy do to long-term interest rates at the zero lower bound?', The Economic Journal 122, 447-66.

Xiong, W. \& Yan, H. (2010), 'Heterogenous expectations and bond markets', Review of Financial Studies 23, 1433-1466. 